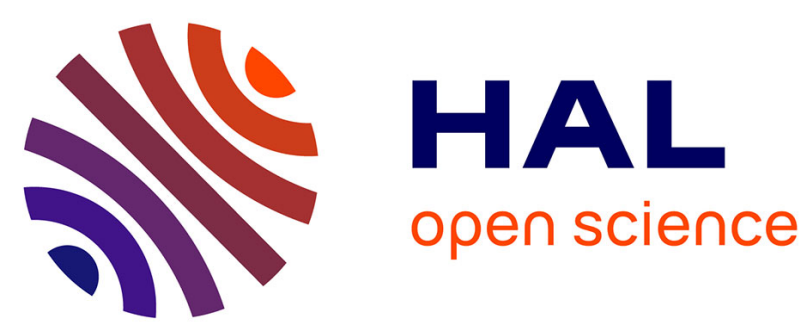

\title{
Speckle observations with PISCO in Merate (Italy): XIV. Astrometric measurements of visual binaries in 2013, and new orbits for ADS 1097, 5871, 7203, 7775, 9378, 9578 and 11186
}

M. Scardia, Jean-Louis Prieur, L. Pansecchi, R.W. Argyle, A. Zanutta, E. Aristidi

\section{To cite this version:}

M. Scardia, Jean-Louis Prieur, L. Pansecchi, R.W. Argyle, A. Zanutta, et al.. Speckle observations with PISCO in Merate (Italy): XIV. Astrometric measurements of visual binaries in 2013, and new orbits for ADS 1097, 5871, 7203, 7775, 9378, 9578 and 11186. Astronomical Notes / Astronomische Nachrichten, 2014, 000 (8), pp.1 - 14. 10.1002/asna.201412054 . hal-02357176

\author{
HAL Id: hal-02357176 \\ https://hal.science/hal-02357176
}

Submitted on 9 Nov 2019

HAL is a multi-disciplinary open access archive for the deposit and dissemination of scientific research documents, whether they are published or not. The documents may come from teaching and research institutions in France or abroad, or from public or private research centers.
L'archive ouverte pluridisciplinaire HAL, est destinée au dépôt et à la diffusion de documents scientifiques de niveau recherche, publiés ou non, émanant des établissements d'enseignement et de recherche français ou étrangers, des laboratoires publics ou privés. 


\title{
Speckle observations with PISCO in Merate (Italy): XIV. Astrometric measurements of visual binaries in 2013, and new orbits for ADS 1097, 5871, 7203, 7775, 9378, 9578 and 11186
}

\author{
M. Scardia ${ }^{1}$, J.-L. Prieur ${ }^{2,3}$, L. Pansecchi ${ }^{1}$, R.W. Argyle ${ }^{4}$, A. Zanutta ${ }^{1}$, and E. Aristidi ${ }^{5}$ \\ 1 INAF - Osservatorio Astronomico di Brera, Via E. Bianchi 46, 23807 Merate, Italy \\ ${ }^{2}$ Université de Toulouse - UPS-OMP - IRAP, Toulouse, France \\ 3 CNRS - IRAP, 14 avenue Edouard Belin, 31400 Toulouse, France \\ 4 Institute of Astronomy, Madingley Road, Cambridge, CB3 0HA, United Kingdom \\ 5 Université de Nice-Sophia Antipolis -Laboratoire Lagrange-CNRS-OCA, Parc Valrose 06108 Nice cedex 2, \\ France
}

Received March 20, 2015; accepted

\begin{abstract}
Key words Stars: binaries: close - binaries: visual — astrometry - techniques: interferometric - stars: individual (ADS 1097, ADS 5871, ADS 7203, ADS 7775, ADS 9378, ADS 9578 and ADS 11186)

We present relative astrometric measurements of visual binaries, made in 2013 with the speckle camera PISCO at the 102-cm Zeiss telescope of Brera Astronomical Observatory, in Merate. Our observing list contains orbital couples as well as binaries whose motion is still uncertain. We obtained 134 new measurements of 129 visual binary stars, with angular separations in the range $0^{\prime \prime} .23-5^{\prime \prime}$, and an average accuracy of $0^{\prime \prime} .01$. The mean error on the position angles is $0^{\circ} .5$. Most of the position angles were determined without the usual $180^{\circ}$ ambiguity with the application of triple-correlation techniques and/or by inspection of the long integration files. We also present new revised orbits for ADS 1097, 5871, 7203, 7775, 9378, 9578 and 11186, partly derived from PISCO observations. The corresponding estimated values for the masses of those systems are compatible with the spectral types.
\end{abstract}

\section{Introduction}

This paper presents the results of speckle observations of visual binary stars made in Merate (Italy) in 2013 with the Pupil Interferometry Speckle camera and COronagraph (PISCO) on the 102-cm Zeiss telescope of INAF - Osservatorio Astronomico di Brera (OAB, Brera Astronomical Observatory). It is the fourteenth of a series whose purpose is to contribute to the determination of binary orbits (Scardia et al. 2005, 2006, 2007, 2008a, Prieur et al. 2008, Scardia et al. 2009, Prieur et al. 2009, Scardia et al. 2010, Prieur et al. 2010, Scardia et al. 2011, Prieur et al. 2012, Scardia et al. 2013, and Prieur et al. 2014 herein: Papers I to XIII). The focal instrument PISCO was developed at Observatoire Midi-Pyrénées (France) and first used at Pic du Midi from 1993 to 1998. It was moved to Merate in 2003 and installed on the INAF Zeiss telescope that has been dedicated to binary star observations since that epoch.

In Sect. 2, we briefly describe our observations. In Sect. 3, we present and discuss the astrometric measurements. We also compare those measurements with the ephemerides computed with the published orbital elements, when available. Finally in Sect. 4 we present the new revised orbits that we have computed for ADS 1097, 5871, 7203, 7775, 9378, 9578 and 11186 and discuss the estimated values for the masses of those systems.

\section{Observations}

The observations were carried out with the PISCO speckle camera and the ICCD (Intensified Charge Coupled Device) detector belonging to Nice University (France). This instrumentation is presented in Prieur et al. (1998) and our observing procedure is described in detail in Paper VI.

\subsection{Observing list}

Our observing list basically includes all the visual binaries for which new measurements are needed to improve their orbits, that are accessible with our instrumentation. It consists of a few thousands objects. A detailed description can be found in our previous papers (e.g., Paper VI).

The distribution of the angular separations measured in this paper is displayed in Fig. 1a and shows a maximum for $\rho \approx 0^{\prime \prime} .6$. The largest separation of $4^{\prime \prime} .98$ 

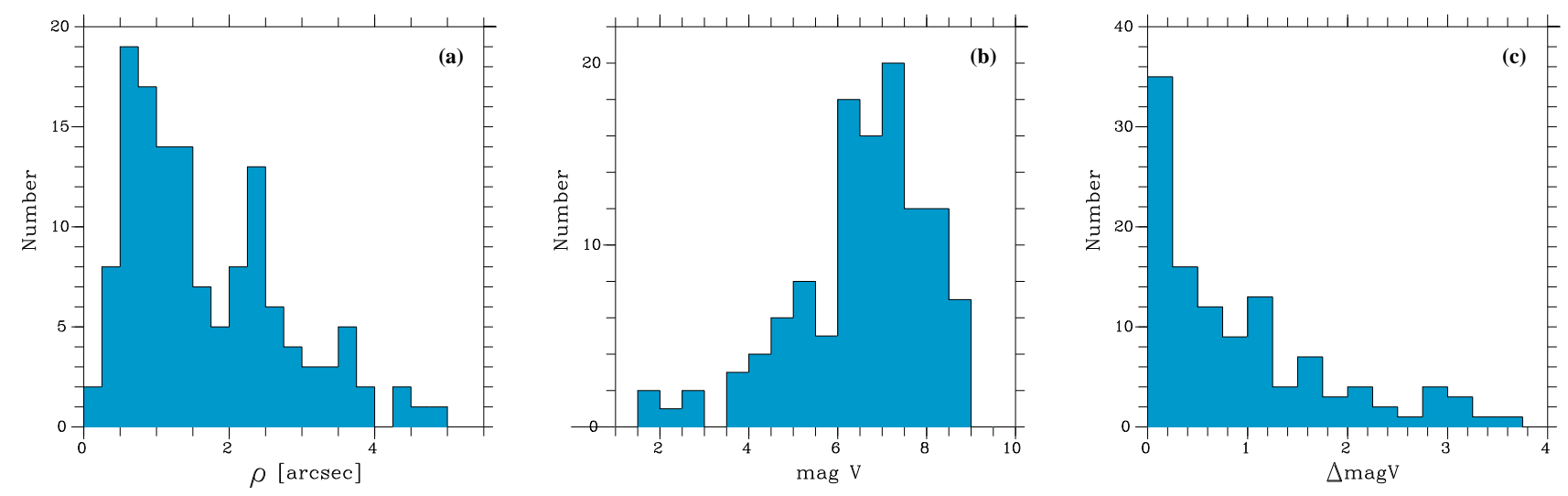

Fig. 1 Distribution of the angular separations of the 134 measurements of Table 1 (a), the total visual magnitudes of the corresponding binaries (b) and the differences of magnitude between their two components (c).

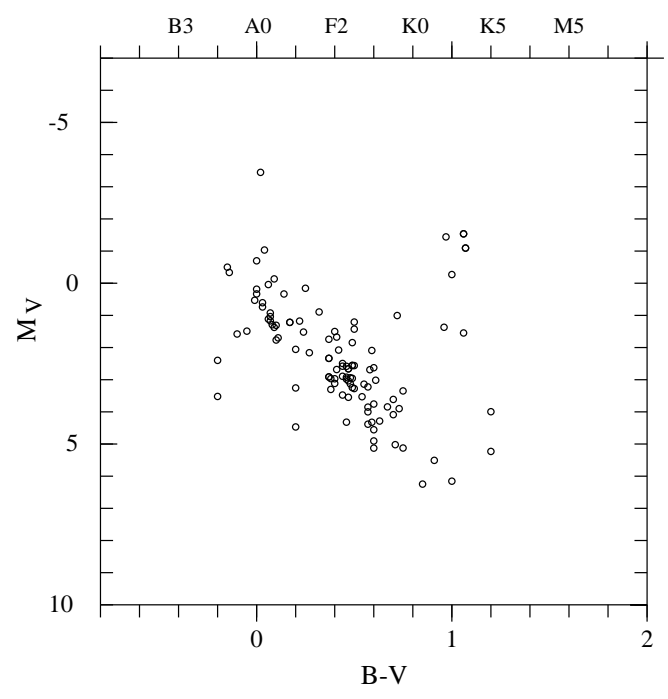

Fig. 2 HR diagram of the binaries measured in Table 1, for which Hipparcos parallaxes were obtained with a relative error smaller than $50 \%$ (i.e., 109 objects).

was obtained for ADS 6175 and the smallest separation was measured for $\operatorname{ADS} 11468$, with $\rho=0^{\prime \prime} .228$. Let us recall that the diffraction limit is $\rho_{d}=\lambda / D \approx 0^{\prime \prime} .13$ for the Zeiss telescope (aperture $D=1.02 \mathrm{~m}$ ) and the $R$ filter $(\lambda=650 \mathrm{~nm})$.

The distribution of the apparent magnitudes $m_{V}$ and of the difference of magnitudes $\Delta m_{V}$ between the two components are plotted in Figs. $1 \mathrm{~b}$ and 1c, respectively. The telescope aperture and detector sensitivity led to a limiting magnitude of about $m_{V}=9$ (Fig. 1b) and a maximum $\Delta m_{V}$ for speckle measurements of about 3.8 (Fig. 1c).

Using the Hipparcos parallaxes, we were able to construct the HR diagram of those binaries, which is displayed in Fig. 2. We only plotted the objects for which the relative uncertainty on the parallax was smaller than 50\%. This concerned 109 objects only.

\section{Astrometric measurements}

The 134 astrometric measurements obtained with the observations made in 2013 are displayed in Table 1. They concern 129 visual binaries. For each object, we report its WDS name (Washington Double Star Catalog, Mason et al. 2014, hereafter WDS catalog) in Col. 1, the official double star designation in Col. 2 (sequence is "discoverer-number"), and the ADS number in Col. 3 (Aitken, 1932) when available. For each observation, we then give the epoch in Besselian years (Col. 4), the filter (Col. 5), the focal length of the eyepiece used for magnifying the image (Col. 6), the angular separation $\rho$ (Col. 7) with its error (Col. 8) in arcseconds, and the position angle $\theta$ (Col. 9) with its error (Col. 10) in degrees. In Col. 11, we report some notes and some information about the secondary peaks of the auto-correlation files (e.g. diffuse, faint or elongated) or about the power spectrum (NF: no fringes). We reported "Triple?" for ADS 9630, since we noticed the presence of a faint pattern in the secondary peaks of the autocorrelation that may be due to the presence of an unknown third component. For the systems with a known orbit, the $(O-C)$ (Observed minus Computed) residuals of the $\rho$ and $\theta$ measurements are displayed in Cols. 13 and 14, respectively. The corresponding authors are given in Col. 12, using the bibliographic style of the "Sixth Catalog of Orbits of Visual Binary Stars" (Hartkopf \& Mason, 2014, hereafter OC6). When not explicitly specified, the measurements refer to the AB components of those systems. In Col. 14, the symbol ${ }^{Q}$ indicates that there was a quadrant inconsistency between our measures and the positions derived from the orbital elements published for this object.

The characteristics of the $R$ and $R L$ filters used for obtaining those measurements are given in Table 2 of Paper XIII. Some objects were observed without any filter because they were too faint. This is indicated with 
Table 1 Table of speckle measurements and O-C residuals with published orbits (begin.)

\begin{tabular}{|c|c|c|c|c|c|c|c|c|c|c|c|c|}
\hline WDS & Name & ADS & Epoch & Fil. & $\begin{array}{l}\text { Eyep. } \\
(\mathrm{mm})\end{array}$ & $\begin{array}{c}\rho \\
\left({ }^{\prime \prime}\right)\end{array}$ & $\begin{array}{l}\sigma_{\rho} \\
\left(^{\prime \prime}\right)\end{array}$ & $\begin{array}{cc}\theta & \sigma_{\theta} \\
\left({ }^{\circ}\right) & \left(^{\circ}\right)\end{array}$ & Notes & Orbit & $\begin{array}{r}\Delta \rho(\mathrm{O}-\mathrm{C}) \\
\quad\left({ }^{\prime \prime}\right)\end{array}$ & $\begin{array}{r}\Delta \theta(\mathrm{O}-\mathrm{C}) \\
\left({ }^{\circ}\right)\end{array}$ \\
\hline $05247+6323$ & STF677 & 3956 & 2013.174 & $\mathrm{R}$ & 20 & 1.116 & 0.017 & $116.4^{*} 0.6$ & & Hrt2008 & -0.02 & 1.0 \\
\hline $05308+0557$ & STF728 & 4115 & 2013.174 & $\mathrm{R}$ & 20 & 1.277 & 0.008 & $44.9^{*} 0.4$ & & USN1999b & -0.02 & 0.3 \\
\hline $05312+0318$ & STF729 & 4123 & 2013.174 & $\mathrm{R}$ & 20 & 1.922 & 0.010 & $27.2^{*} 0.4$ & & & & \\
\hline $07057+5245$ & STF1009 & 5746 & 2013.174 & $\mathrm{R}$ & 32 & 4.306 & 0.022 & $147.7^{*} 0.3$ & & & & \\
\hline $07128+2713$ & STF1037 & 5871 & 2013.174 & $\mathrm{R}$ & 20 & 0.978 & 0.011 & $307.2^{*} 0.5$ & & Sta1981a & 0.00 & 0.6 \\
\hline$"$ & $"$ & $"$ & $"$ & $"$ & $"$ & $"$ & $"$ & $" \quad "$ & & This paper & 0.04 & 0.3 \\
\hline $07346+3153$ & STF1110 & 6175 & 2013.174 & $\mathrm{R}$ & 32 & 4.983 & 0.025 & $55.4^{*} 0.3$ & & Hei1988a & 0.06 & -0.5 \\
\hline $08095+3213$ & STF1187 & 6623 & 2013.289 & $\mathrm{R}$ & 32 & 3.127 & 0.016 & $21.1^{*} 0.3$ & & Ole2001 & 0.18 & 0.4 \\
\hline $08482+0235$ & BU335 & 7003 & 2013.174 & $\mathrm{R}$ & 32 & 2.485 & 0.021 & $264.9^{*} 0.5$ & $\mathrm{NF}$ & & & \\
\hline $08500+3935$ & STF1279 & 7019 & 2013.297 & $\mathrm{R}$ & 32 & 1.253 & 0.016 & $87.0^{*} 0.3$ & & & & \\
\hline $08505+2308$ & AG157 & 7035 & 2013.297 & W & 32 & 2.272 & 0.016 & $75.2^{*} 0.4$ & Elongated & & & \\
\hline $08561+4341$ & STF3120 & 7092 & 2013.175 & $\mathrm{R}$ & 32 & 1.343 & 0.016 & $0.2^{*} 0.6$ & & & & \\
\hline $08571+1045$ & A2968 & 7102 & 2013.297 & W & 20 & 1.164 & 0.011 & $132.8^{*} 0.4$ & Elongated & & & \\
\hline $09006+4147$ & KUI37 & - & 2013.289 & $\mathrm{R}$ & 10 & 0.553 & 0.005 & $215.0^{*} 0.4$ & Elongated & Sod1999 & -0.01 & -0.8 \\
\hline $09104+6708$ & STF1306 & 7203 & 2013.287 & $\mathrm{R}$ & 20 & 4.339 & 0.022 & $348.5^{*} 0.3$ & $\mathrm{NF}$ & Sca1985c & 0.09 & -0.4 \\
\hline$"$ & & $"$ & $"$ & $"$ & $"$ & $"$ & $"$ & $" \quad "$ & & This paper & 0.06 & -0.1 \\
\hline $09127+1632$ & STF1322 & 7236 & 2013.289 & $\mathrm{R}$ & 32 & 1.801 & 0.016 & $53.0^{*} 0.4$ & & & & \\
\hline $09136+4659$ & STF1318 & 7243 & 2013.289 & $\mathrm{R}$ & 32 & 2.626 & 0.017 & $228.3^{*} 0.4$ & & & & \\
\hline $09188+3648$ & STF1334 & 7292 & 2013.287 & $\mathrm{R}$ & 20 & 2.641 & 0.013 & $223.2^{*} 0.3$ & & & & \\
\hline $09210+3811$ & STF1338 & 7307 & 2013.314 & $\mathrm{R}$ & 20 & 1.116 & 0.008 & $305.9^{*} 0.4$ & & Sca2002b & -0.02 & 0.2 \\
\hline $09239+2754$ & STT201 & 7344 & 2013.314 & $\mathrm{R}$ & 32 & 1.220 & 0.017 & $204.8^{*} 0.4$ & & & & \\
\hline $09245+1808$ & A 2477 & 7341 & 2013.314 & $\mathrm{R}$ & 20 & 0.452 & 0.008 & $3.4^{*} 1.2$ & Elongated & FMR2014a & 0.02 & 0.9 \\
\hline $09285+0903$ & STF1356 & 7390 & 2013.289 & $\mathrm{R}$ & 20 & 0.780 & 0.008 & $107.0^{*} 0.3$ & & Mut2010b & -0.01 & -0.2 \\
\hline $09300+4216$ & A1985 & 7398 & 2013.289 & $\mathrm{R}$ & 32 & 1.577 & 0.016 & $24.6 \quad 0.6$ & & & & \\
\hline $09377+1153$ & HJ165 & 7461 & 2013.314 & $\mathrm{R}$ & 32 & 2.361 & 0.027 & $347.8^{*} 0.4$ & & & & \\
\hline $09414+3857$ & STF1374 & 7477 & 2013.289 & $\mathrm{R}$ & 32 & 2.793 & 0.016 & $308.9^{*} 0.3$ & & Lin2013a & -0.03 & -1.2 \\
\hline $09476+5057$ & HU630 & 7514 & 2013.314 & $\mathrm{R}$ & 32 & 2.244 & 0.024 & $\begin{array}{lll}72.6 & 0.4\end{array}$ & & & & \\
\hline $09513+6037$ & STF1381 & 7536 & 2013.289 & $\mathrm{R}$ & 32 & 0.798 & 0.022 & $\begin{array}{lll}186.2 & 0.3\end{array}$ & & & & \\
\hline $09521+5404$ & STT208 & 7545 & 2013.290 & $\mathrm{R}$ & 10 & 0.381 & 0.003 & $300.3 \quad 0.4$ & & Hei1996c & -0.03 & -1.0 \\
\hline $09524+2659$ & STF1389 & 7551 & 2013.314 & $\mathrm{R}$ & 32 & 2.481 & 0.019 & $289.8^{*} 0.3$ & & & & \\
\hline $09572+4554$ & STF1394 & 7588 & 2013.314 & $\mathrm{R}$ & 32 & 4.593 & 0.025 & $249.2^{*} 0.3$ & & & & \\
\hline $10029+0742$ & STF1403 & 7617 & 2013.314 & W & 32 & 2.876 & 0.027 & $333.5^{*} 0.3$ & & & & \\
\hline $10163+1744$ & STT215 & 7704 & 2013.298 & $\mathrm{R}$ & 20 & 1.485 & 0.008 & $176.8^{*} 0.3$ & & Wrz1956c & -0.07 & -0.4 \\
\hline $10205+0626$ & STF1426 & 7730 & 2013.298 & $\mathrm{R}$ & 20 & 0.910 & 0.012 & $311.7^{*} 0.3$ & Diffuse & Sca2008c & 0.01 & -0.6 \\
\hline$"$ & & & $"$ & $"$ & $"$ & $"$ & $"$ & $" \quad "$ & & Nov2006 & -0.00 & -0.5 \\
\hline $10227+1521$ & STT216 & 7744 & 2013.298 & $\mathrm{R}$ & 32 & 2.266 & 0.027 & $232.2^{*} 0.3$ & & Sca2009c & 0.06 & 0.0 \\
\hline $10250+2437$ & STF1429 & 7758 & 2013.298 & $\mathrm{R}$ & 20 & 0.809 & 0.013 & $156.9^{*} 0.3$ & & Zul1981 & 0.06 & -1.0 \\
\hline $10350+0839$ & STF1450 & 7837 & 2013.298 & $\mathrm{R}$ & 32 & 2.108 & 0.017 & $155.8^{*} 0.3$ & & & & \\
\hline $10406+4209$ & STF1460 & 7878 & 2013.298 & $\mathrm{R}$ & 32 & 3.760 & 0.019 & $162.0^{*} 0.3$ & & & & \\
\hline $11037+6145$ & BU1077 & 8035 & 2013.287 & V & 10 & 0.698 & 0.003 & $0.1^{*} 0.3$ & & Sca2011a & 0.01 & -1.7 \\
\hline$"$ & $"$ & & 2013.366 & $\mathrm{R}$ & 10 & 0.682 & 0.007 & $359.8^{*} 0.5$ & $\mathrm{NF}$ & Sca2011a & -0.01 & -1.6 \\
\hline $11050+3825$ & HO378 & 8047 & 2013.287 & $\mathrm{R}$ & 20 & 1.083 & 0.011 & $236.1^{*} 1.0$ & & & & \\
\hline $11136+5525$ & A1353 & 8092 & 2013.287 & $\mathrm{R}$ & 20 & 0.569 & 0.008 & $209.8^{*} 0.7$ & & Doc1999b & 0.03 & 0.1 \\
\hline $11137+2008$ & STF1517 & 8094 & 2013.287 & $\mathrm{R}$ & 20 & 0.657 & 0.008 & $\begin{array}{ll}316.1 & 0.4\end{array}$ & & FMR2011a & -0.02 & -0.5 \\
\hline $11154+2734$ & STF1521 & 8105 & 2013.314 & $\mathrm{R}$ & 32 & 3.650 & 0.018 & $96.3^{*} 0.3$ & & & & \\
\hline $11182+3132$ & STF1523 & 8119 & 2013.314 & $\mathrm{R}$ & 20 & 1.657 & 0.010 & $187.7^{*} 0.3$ & & Msn1995 & -0.02 & 0.0 \\
\hline $11190+1416$ & STF1527 & 8128 & 2013.287 & $\mathrm{R}$ & 10 & 0.300 & 0.006 & $223.8 \quad 0.3$ & Elongated & Tok2012b & -0.01 & 1.0 \\
\hline $11239+1032$ & STF1536 & 8148 & 2013.314 & $\mathrm{R}$ & 20 & 2.070 & 0.014 & $97.8^{*} 0.3$ & $\mathrm{NF}$ & Sta1980a & 0.02 & -0.1 \\
\hline $11245+2037$ & STF1537 & 8149 & 2013.290 & $\mathrm{R}$ & 32 & 2.277 & 0.016 & $358.3^{*} 0.7$ & & & & \\
\hline $11332+4927$ & HU727 & 8210 & 2013.314 & $\mathrm{R}$ & 32 & 1.220 & 0.016 & $204.7^{*} 1.0$ & & & & \\
\hline $11363+2747$ & STF1555 & 8231 & 2013.287 & $\mathrm{R}$ & 20 & 0.719 & 0.008 & $148.8^{*} 0.4$ & & Doc2007i & 0.04 & -1.1 \\
\hline $11371+4040$ & A1996 & 8241 & 2013.366 & $\mathrm{R}$ & 32 & 1.991 & 0.036 & $\begin{array}{ll}189.6 & 0.7\end{array}$ & & & & \\
\hline $11388+6421$ & STF1559 & 8249 & 2013.314 & $\mathrm{R}$ & 32 & 1.956 & 0.021 & $322.9^{*} 0.4$ & & & & \\
\hline $12417-0127$ & STF 1670 & 8630 & 2013.448 & RL & 20 & 2.024 & 0.010 & $9.7^{*} 0.4$ & & Sca2007c & -0.00 & -0.1 \\
\hline$"$ & $"$ & & 2013.459 & $\mathrm{R}$ & 20 & 2.021 & 0.010 & $9.6^{*} 0.3$ & & Sca2007c & -0.01 & -0.2 \\
\hline
\end{tabular}


Table 1 Table of speckle measurements and O-C residuals with published orbits (cont.)

\begin{tabular}{|c|c|c|c|c|c|c|c|c|c|c|c|c|c|}
\hline WDS & Name & ADS & Epoch & Fil. & $\begin{array}{l}\text { Eyep. } \\
(\mathrm{mm})\end{array}$ & $\underset{\rho}{\rho}$ & $\begin{array}{l}\sigma_{\rho} \\
\left({ }^{\prime \prime}\right)\end{array}$ & $\begin{array}{c}\theta \\
\left({ }^{\circ}\right)\end{array}$ & $\begin{array}{l}\sigma_{\theta} \\
\left(^{\circ}\right)\end{array}$ & Notes & Orbit & $\begin{array}{r}\Delta \rho(\mathrm{O}-\mathrm{C}) \\
\left({ }^{\prime \prime}\right)\end{array}$ & $\begin{array}{r}\Delta \theta(\mathrm{O}-\mathrm{C}) \\
\left(^{\circ}\right)\end{array}$ \\
\hline $13007+5622$ & BU1082 & 8739 & 2013.448 & RL & 20 & 0.918 & 0.015 & $111.4^{*}$ & * 0.3 & & Sca2012c & -0.06 & 1.2 \\
\hline$"$ & $"$ & $"$ & $"$ & $"$ & $"$ & $"$ & $"$ & $"$ & $"$ & & Doc2014 & -0.00 & 0.5 \\
\hline$"$ & $"$ & $"$ & $"$ & $"$ & $"$ & $"$ & $"$ & $"$ & $"$ & & Dru2014 & 0.00 & -0.8 \\
\hline$"$ & $"$ & $"$ & 2013.459 & $\mathrm{RL}$ & 20 & 0.940 & 0.011 & $111.5^{*}$ & * 0.3 & & Sca2012c & -0.03 & 1.3 \\
\hline$"$ & $"$ & $"$ & $"$ & $"$ & $"$ & $"$ & $"$ & $"$ & $"$ & & Doc2014 & 0.02 & 0.6 \\
\hline$"$ & $"$ & $"$ & $"$ & $"$ & $"$ & $"$ & $"$ & $"$ & $"$ & & Dru2014 & 0.03 & -0.7 \\
\hline $13048+7302$ & BU799 & 8772 & 2013.459 & $\mathrm{R}$ & 20 & 1.387 & 0.008 & $265.1^{*}$ & * 0.3 & & & & \\
\hline $13100+1732$ & STF1728 & 8804 & 2013.459 & $\mathrm{R}$ & 10 & 0.346 & 0.004 & 11.8 & 0.5 & Elongated & Pal2005b & -0.00 & $0.0^{Q}$ \\
\hline $13120+3205$ & STT261 & 8814 & 2013.462 & $\mathrm{R}$ & 20 & 2.613 & 0.013 & $338.3^{*}$ & * 0.3 & & Kis2012 & 0.04 & -0.2 \\
\hline $13207+0257$ & STF1734 & 8864 & 2013.462 & $\mathrm{R}$ & 20 & 1.112 & 0.008 & $174.0^{*}$ & * 0.3 & & & & \\
\hline $14153+0308$ & STF1819 & 9182 & 2013.448 & $\mathrm{R}$ & 20 & 0.877 & 0.008 & $171.6^{*}$ & * 0.6 & & Sca2012b & -0.01 & 0.6 \\
\hline $14203+4830$ & STF1834 & 9229 & 2013.459 & $\mathrm{R}$ & 20 & 1.641 & 0.008 & $102.6^{*}$ & * 0.3 & & USN2000c & 0.07 & -1.0 \\
\hline $14220+5107$ & A148 & 9238 & 2013.459 & $\mathrm{R}$ & 20 & 0.553 & 0.008 & 191.6 & 1.4 & NF & & & \\
\hline $14336+3535$ & STF1858 & 9312 & 2013.497 & $\mathrm{R}$ & 32 & 3.046 & 0.016 & $37.9^{*}$ & * 0.3 & & & & \\
\hline $14380+5135$ & STF1863 & 9329 & 2013.459 & $\mathrm{R}$ & 20 & 0.648 & 0.008 & $59.2^{*}$ & * 0.3 & & Zir2013a & -0.00 & -1.3 \\
\hline $14407+3117$ & STF1867 & 9340 & 2013.459 & $\mathrm{R}$ & 20 & 0.703 & 0.008 & $353.7^{*}$ & $* 0.5$ & & Zir2013a & 0.03 & -0.4 \\
\hline $14450+2704$ & STF1877 & 9372 & 2013.459 & $\mathrm{R}$ & 20 & 2.855 & 0.014 & $343.7^{*}$ & * 0.3 & & & & \\
\hline $14455+4223$ & STT285 & 9378 & 2013.448 & $\mathrm{R}$ & 20 & 0.464 & 0.019 & $85.4^{*}$ & * 1.1 & & Cou1973b & -0.03 & 1.6 \\
\hline$"$ & $"$ & $"$ & $"$ & $"$ & $"$ & $"$ & $"$ & $"$ & $"$ & & This paper & -0.02 & 1.5 \\
\hline$"$ & $"$ & $"$ & 2013.462 & $\mathrm{R}$ & 20 & 0.498 & 0.008 & $83.6^{*}$ & * 0.9 & & Cou1973b & 0.00 & -0.2 \\
\hline$"$ & $"$ & $"$ & $"$ & $"$ & $"$ & $"$ & $"$ & $"$ & $"$ & & This paper & 0.02 & -0.2 \\
\hline $14489+0557$ & STF1883 & 9392 & 2013.459 & $\mathrm{R}$ & 20 & 0.983 & 0.008 & 278.2 & 0.4 & & Sta1982b & -0.08 & 0.2 \\
\hline $15183+2650$ & STF1932 & 9578 & 2013.448 & $\mathrm{R}$ & 20 & 1.620 & 0.008 & $264.2^{*}$ & * 0.3 & & Sod1999 & -0.01 & 0.2 \\
\hline$"$ & $"$ & $"$ & $"$ & $"$ & $"$ & $"$ & $"$ & $"$ & $"$ & & This paper & -0.00 & -0.4 \\
\hline $15257+2638$ & STF1941 & 9630 & 2013.448 & $\mathrm{R}$ & 32 & 1.367 & 0.025 & 211.1 & 1.1 & Triple? & & & \\
\hline $15277+0606$ & STF1944 & 9647 & 2013.448 & $\mathrm{~W}$ & 20 & 0.657 & 0.008 & $295.2^{*}$ & * 0.3 & & & & \\
\hline $15348+1032$ & STF1954 & 9701 & 2013.459 & $\mathrm{R}$ & 32 & 3.985 & 0.027 & $172.0^{*}$ & * 0.3 & & Kiy2010 & -0.08 & 0.3 \\
\hline $15360+3948$ & STT298 & 9716 & 2013.459 & $\mathrm{R}$ & 20 & 1.160 & 0.008 & $182.4^{*}$ & * 0.4 & & Cou1989c & -0.02 & 0.2 \\
\hline $16289+1825$ & STF2052 & 10075 & 2013.672 & $\mathrm{R}$ & 32 & 2.307 & 0.016 & $119.2^{*}$ & * 0.3 & & Sca1984d & -0.03 & -0.7 \\
\hline $16518+2840$ & STF2107 & 10235 & 2013.678 & $\mathrm{R}$ & 20 & 1.416 & 0.014 & $103.2^{*}$ & * 0.3 & & Sca2003c & 0.02 & -1.1 \\
\hline $16564+6502$ & STF2118 & 10279 & 2013.675 & $\mathrm{R}$ & 20 & 1.003 & 0.008 & $65.1^{*}$ & * 0.3 & & Sca2002d & -0.15 & -1.7 \\
\hline $17020+0827$ & STF2114 & 10312 & 2013.675 & $\mathrm{R}$ & 20 & 1.310 & 0.009 & $195.3^{*}$ & * 0.3 & & & & \\
\hline $17053+5428$ & STF2130 & 10345 & 2013.672 & $\mathrm{R}$ & 20 & 2.444 & 0.012 & 4.1 & 0.3 & & Pru2012 & -0.03 & -0.1 \\
\hline $17055+1033$ & BU357 & 10336 & 2013.675 & $\mathrm{RL}$ & 32 & 1.389 & 0.016 & $305.7^{*}$ & * 0.8 & Faint & & & \\
\hline $17246+1536$ & STF2160 & 10528 & 2013.683 & $\mathrm{R}$ & 32 & 3.740 & 0.024 & $66.3^{*}$ & * 0.5 & $\mathrm{NF}$ & & & \\
\hline $17266+3546$ & STF2168 & 10558 & 2013.684 & $\mathrm{R}$ & 32 & 2.312 & 0.016 & $201.9^{*}$ & * 0.6 & & & & \\
\hline $17304-0104$ & STF2173 & 10598 & 2013.684 & $\mathrm{R}$ & 20 & 0.792 & 0.013 & $147.9^{*}$ & * 0.3 & & Hei1994a & -0.02 & -0.9 \\
\hline $17317+1111$ & AG354 & 10612 & 2013.722 & $\mathrm{R}$ & 32 & 2.522 & 0.016 & $51.0^{*}$ & * 0.3 & & & & \\
\hline $17350+6153$ & BU962 & 10660 & 2013.722 & $\mathrm{R}$ & 20 & 0.625 & 0.029 & $300.5^{*}$ & * 1.0 & & Sod1999 & -0.01 & -2.1 \\
\hline $17457+1743$ & STF2205 & 10769 & 2013.730 & $\mathrm{R}$ & 32 & 0.931 & 0.016 & $7.3^{*}$ & * 0.9 & & Cve2008a & -0.08 & 0.5 \\
\hline $17471+1742$ & STF2215 & 10795 & 2013.678 & $\mathrm{R}$ & 10 & 0.470 & 0.005 & $251.4^{*}$ & * 0.6 & Elongated & Cve2006e & 0.01 & 2.5 \\
\hline $17479+1449$ & STF2222 & 10803 & 2013.733 & $\mathrm{R}$ & 32 & 2.263 & 0.041 & $62.5^{*}$ & * 0.5 & & & & \\
\hline $17506+0714$ & STT337 & 10828 & 2013.678 & $\mathrm{R}$ & 20 & 0.556 & 0.016 & 164.4 & 0.7 & & Doc1990a & 0.04 & 0.6 \\
\hline $17512+4454$ & STF2242 & 10849 & 2013.678 & $\mathrm{R}$ & 32 & 3.252 & 0.016 & $145.4^{*}$ & * 0.4 & & & & \\
\hline $17518+2814$ & STF2239 & 10851 & 2013.678 & $\mathrm{R}$ & 32 & 2.444 & 0.016 & $317.0^{*}$ & * 0.5 & & & & \\
\hline $17520+1520$ & STT338 & 10850 & 2013.678 & $\mathrm{R}$ & 20 & 0.807 & 0.008 & $163.6^{*}$ & * 0.4 & & Pru2012 & -0.02 & -0.8 \\
\hline $17567+4837$ & STF2258 & 10924 & 2013.730 & $\mathrm{R}$ & 32 & 2.075 & 0.024 & $220.9^{*}$ & $* * 1.1$ & & & & \\
\hline $17571+0004$ & STF2244 & 10912 & 2013.681 & $\mathrm{R}$ & 12 & 0.681 & 0.011 & $99.1^{*}$ & * 0.6 & Elongated & Hei1997 & 0.15 & -2.1 \\
\hline $18014+6557$ & STF2284 & 11016 & 2013.675 & $\mathrm{R}$ & 32 & 3.547 & 0.025 & $191.1^{*}$ & * 0.3 & & & & \\
\hline $18101+1629$ & STF2289 & 11123 & 2013.675 & $\mathrm{R}$ & 20 & 1.206 & 0.008 & $218.6^{*}$ & $* 0.5$ & & Hop1964b & -0.03 & 2.7 \\
\hline $18146+0011$ & STF2294 & 11186 & 2013.675 & $\mathrm{R}$ & 20 & 1.335 & 0.008 & $92.5^{*}$ & * 0.3 & & Luy1934a & 0.17 & -0.3 \\
\hline$"$ & $"$ & $"$ & $"$ & $"$ & $"$ & $"$ & $"$ & $"$ & $"$ & & This paper & 0.02 & -0.9 \\
\hline $18239+5848$ & STF2323 & 11336 & 2013.722 & $\mathrm{R}$ & 32 & 3.656 & 0.018 & $348.1^{*}$ & * 0.3 & & Nov2006e & -0.09 & -0.2 \\
\hline $18250+2724$ & STF2315 & 11334 & 2013.681 & $\mathrm{R}$ & 12 & 0.648 & 0.006 & $118.1^{*}$ & * 0.3 & & WSI2004b & 0.03 & 0.6 \\
\hline $18278+2442$ & STF2320 & 11373 & 2013.733 & $\mathrm{R}$ & 32 & 1.038 & 0.025 & $358.4^{*}$ & * 0.6 & & & & \\
\hline $18320+0647$ & STT354 & 11432 & 2013.684 & $\mathrm{R}$ & 20 & 0.562 & 0.008 & $214.9^{*}$ & * 1.4 & & Zir2013a & -0.02 & 1.9 \\
\hline
\end{tabular}


Table 1 Table of speckle measurements and O-C residuals with published orbits (cont.)

\begin{tabular}{|c|c|c|c|c|c|c|c|c|c|c|c|c|}
\hline WDS & Name & $\mathrm{ADS}$ & Epoch & Fil. & $\begin{array}{l}\text { Eyep. } \\
\text { (mm) }\end{array}$ & $\begin{array}{c}\rho \\
\left({ }^{\prime \prime}\right)\end{array}$ & $\begin{array}{l}\sigma_{\rho} \\
\left({ }^{\prime \prime}\right)\end{array}$ & $\begin{array}{cc}\theta & \sigma_{\theta} \\
\left(^{\circ}\right) & \left(^{\circ}\right)\end{array}$ & Notes & Orbit & $\begin{array}{r}\Delta \rho(\mathrm{O}-\mathrm{C}) \\
\left({ }^{\prime \prime}\right)\end{array}$ & $\begin{array}{r}\Delta \theta(\mathrm{O}-\mathrm{C}) \\
\left({ }^{\circ}\right)\end{array}$ \\
\hline $8339+5221$ & A 1377 & 11468 & 2013.675 & $\mathrm{R}$ & 10 & 0.228 & 0.005 & $129.7^{*} 0.5$ & & Mut2010e & -0.02 & 0.5 \\
\hline$"$ & $"$ & & 2013.681 & $\mathrm{R}$ & 12 & 0.228 & 0.009 & $131.3^{*} 1.3$ & & Mut2010e & -0.02 & 2.1 \\
\hline $18355+2336$ & STT359 & 11479 & 2013.675 & $\mathrm{R}$ & 10 & 0.725 & 0.005 & $4.4^{*} 0.4$ & Elongated & Sca2009a & -0.02 & -0.0 \\
\hline $18359+1659$ & STT358 & 11483 & 2013.684 & $\mathrm{R}$ & 20 & 1.623 & 0.008 & $148.6^{*} 0.3$ & & Hei1995 & 0.11 & 1.4 \\
\hline $18443+3940$ & STF2382 & 11635 & 2013.676 & $\mathrm{R}$ & 20 & 2.260 & 0.012 & $346.3^{*} 0.3$ & & Nov2006e & -0.03 & -0.1 \\
\hline$"$ & $"$ & & $"$ & $"$ & $"$ & $"$ & $"$ & $" "$ & & WSI2004b & -0.10 & -0.1 \\
\hline $18443+3940$ & STF2383CD & 11635 & 2013.676 & $\mathrm{R}$ & 20 & 2.352 & 0.012 & $76.5^{*} 0.3$ & & Doc1984b & -0.03 & -0.1 \\
\hline $18508+1059$ & STF2404 & 11750 & 2013.678 & $\mathrm{R}$ & 32 & 3.492 & 0.035 & $180.9^{*} 0.4$ & Diffuse & & & \\
\hline $18540+3723$ & BU137 & 11811 & 2013.678 & $\mathrm{R}$ & 32 & 1.509 & 0.024 & $163.6^{*} 0.4$ & & & & \\
\hline $18545+2037$ & STF2415 & 11816 & 2013.678 & $\mathrm{R}$ & 32 & 1.874 & 0.043 & $287.2^{*} 0.7$ & & & & \\
\hline $18570+3254$ & BU648 & 11871 & 2013.730 & $\mathrm{R}$ & 20 & 1.224 & 0.008 & $245.7^{*} 0.3$ & & Doc2008f & 0.02 & -0.2 \\
\hline $18571+2606$ & STF2422 & 11869 & 2013.678 & $\mathrm{~W}$ & 20 & 0.724 & 0.017 & $69.5^{*} 1.1$ & & & & \\
\hline $18575+5814$ & STF2438 & 11897 & 2013.678 & $\mathrm{R}$ & 20 & 0.850 & 0.008 & $357.9^{*} 0.3$ & & Hrt2001a & 0.02 & 0.6 \\
\hline $18581+4711$ & AG366 & 11899 & 2013.796 & $\mathrm{R}$ & 32 & 1.397 & 0.016 & $187.2^{*} 0.3$ & & & & \\
\hline $19062+3026$ & STF2454 & 12040 & 2013.722 & $\mathrm{R}$ & 32 & 1.253 & 0.016 & $288.1^{*} 0.4$ & & Baz1976 & -0.07 & -0.3 \\
\hline $19159+2727$ & STT371 & 12239 & 2013.796 & $\mathrm{R}$ & 20 & 0.862 & 0.008 & $160.2^{*} 0.3$ & & & & \\
\hline $19160+1610$ & STT368 & 12236 & 2013.796 & $\mathrm{R}$ & 20 & 1.129 & 0.010 & $217.8^{*} 0.8$ & & & & \\
\hline $19177+2302$ & BU248 & 12287 & 2013.733 & $\mathrm{R}$ & 20 & 1.666 & 0.015 & $127.3^{*} 0.3$ & $\mathrm{NF}$ & & & \\
\hline $19266+2719$ & STF2525 & 12447 & 2013.733 & $\mathrm{R}$ & 32 & 2.103 & 0.016 & $288.2^{*} 0.3$ & & Hei1984b & -0.04 & -1.4 \\
\hline $19334+6203$ & STF2553 & 12626 & 2013.730 & $\mathrm{R}$ & 32 & 0.975 & 0.025 & $130.0^{*} 1.3$ & & & & \\
\hline $19346+1808$ & STT375 & 12623 & 2013.730 & $\mathrm{R}$ & 20 & 0.605 & 0.012 & $188.3^{*} 1.3$ & & & & \\
\hline $19365+4101$ & STT378 & 12687 & 2013.730 & $\mathrm{R}$ & 32 & 1.354 & 0.017 & $284.2^{*} 0.3$ & & & & \\
\hline $19450+4508$ & STF2579 & 12880 & 2013.722 & $\mathrm{R}$ & 20 & 2.684 & 0.013 & $217.2^{*} 0.3$ & & Sca2012c & -0.03 & -0.7 \\
\hline $19464+3344$ & STF2576FG & 12889 & 2013.722 & $\mathrm{R}$ & 32 & 2.915 & 0.021 & $157.1^{*} 0.3$ & & & & \\
\hline $19487+3519$ & STT387 & 12972 & 2013.722 & $\mathrm{R}$ & 20 & 0.488 & 0.016 & $113.8^{*} 0.6$ & & WSI2006b & -0.02 & -1.0 \\
\hline $19540+1518$ & STF2596 & 13082 & 2013.722 & $\mathrm{R}$ & 32 & 2.003 & 0.016 & $298.3^{*} 0.3$ & & & & \\
\hline $20014+1045$ & STF2613 & 13256 & 2013.834 & $\mathrm{R}$ & 32 & 3.500 & 0.017 & $354.3^{*} 0.3$ & & Hop1973b & -0.65 & 2.3 \\
\hline $20020+2456$ & STT395 & 13277 & 2013.722 & $\mathrm{R}$ & 20 & 0.774 & 0.008 & $125.1^{*} 0.4$ & & Zir2013a & -0.08 & -1.1 \\
\hline $20102+4357$ & STT400 & 13461 & 2013.834 & $\mathrm{R}$ & 20 & 0.627 & 0.008 & $330.4 \quad 1.5$ & & Hei1997 & -0.01 & -0.6 \\
\hline $20203+3924$ & STF2668AB-C & 13728 & 2013.796 & $\mathrm{R}$ & 32 & 3.334 & 0.017 & $278.5^{*} 0.3$ & & & & \\
\hline $20229+4259$ & HO128 & 13786 & 2013.834 & $\mathrm{R}$ & 20 & 1.335 & 0.008 & $358.5^{*} 0.3$ & & & & \\
\hline $20396+4035$ & STT410 & 14126 & 2013.834 & $\mathrm{R}$ & 20 & 0.856 & 0.009 & $\begin{array}{ll}3.6 & 0.5\end{array}$ & & Hrt2011a & -0.01 & -0.3 \\
\hline $21018+3916$ & STF2746 & 14558 & 2013.722 & $\mathrm{R}$ & 20 & 1.205 & 0.014 & $322.6^{*} 0.3$ & & & & \\
\hline $21103+4359$ & STF2773 & 14711 & 2013.730 & $\mathrm{R}$ & 32 & 3.190 & 0.016 & $112.4^{*} 0.3$ & & & & \\
\hline $21111+4530$ & BRT1146 & - & 2013.730 & $\mathrm{~W}$ & 32 & 2.639 & 0.019 & $345.2^{*} 0.3$ & Elongated & & & \\
\hline
\end{tabular}

Note: In column 9, the exponent ${ }^{*}$ indicates that the position angle $\theta$ could be determined without the $180^{\circ}$ ambiguity. In column 14, the exponent ${ }^{Q}$ indicates discrepant quadrants between our measurements and the published orbits.

$W$ (for "white" light) in the filter column (Col. 5 of Table 1).

As for the other papers of this series, position measurements were obtained by an interactive processing of the auto-correlation files computed in real time during the observations. This processing led to a series of measurements with different background estimates and simulated noise, from which we derived the mean values and the standard deviation of those multiple measurements (see Paper III for more details). The final measures and their errors are displayed in Table 1. The average error values of the measurements reported in this table are $\left\langle\sigma_{\rho}\right\rangle=0^{\prime \prime} .014 \pm 0^{\prime \prime} .007$ and $\left\langle\sigma_{\theta}\right\rangle=0^{\circ} .5 \pm 1^{\circ} .3$.

\subsection{Quadrant determination}

As our astrometric measurements were obtained from the symmetric auto-correlation files, the $\theta$ values first presented a $180^{\circ}$ ambiguity. To resolve this ambiguity and determine the quadrant containing the companion, Aristidi et al. (1997) have proposed a method that can be considered as a restricted triple correlation (RTC hereafter). The quadrants of the measurements indicated in Table 1 were mostly derived from the RTC files that were computed in real time during the observations. However, for the couples with the largest separations, a straightforward determination was done 


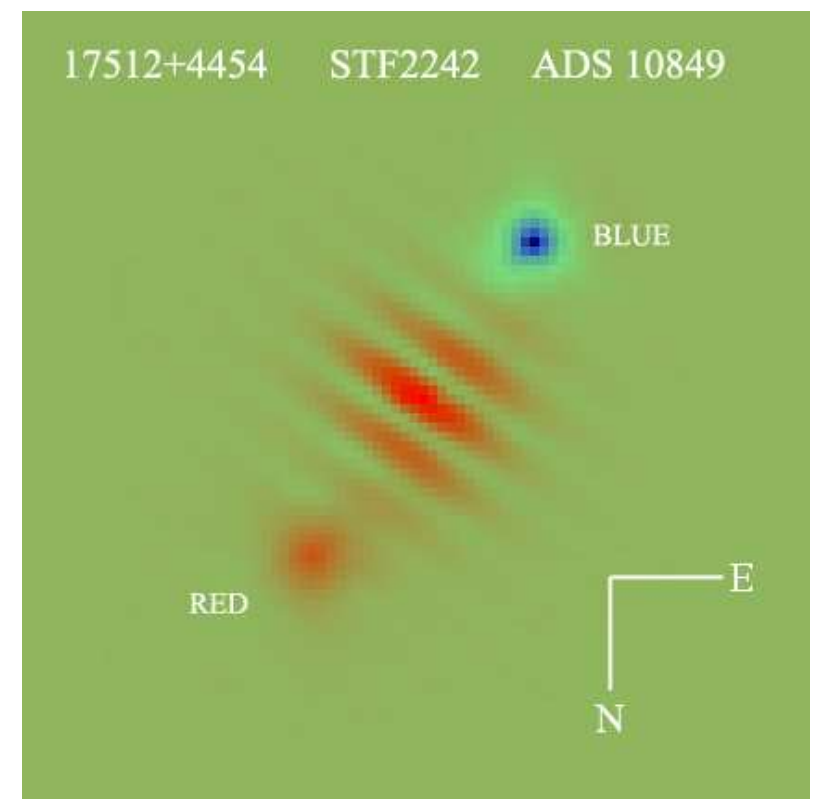

Fig. 3 Quadrant determination of ADS 10849 with a RTC (Restricted Triple Correlation) method. The red and blue spots correspond to the primary and secondary components, respectively. They are labelled RED and BLUE, respectively, for the convenience of the readers of the printed paper (black and white version).

when the companions could be directly spotted on the long integration files.

As a result, in Table 1, we are able to give the unambiguous (i.e. "absolute") position angles of 119 out of 134 measurements, i.e. $89 \%$ of the total. They are marked with an asterisk in Col 9. When our quadrant determination procedure failed, the angular measurement was reduced to the quadrant reported in the WDS catalog, which is extracted from the Fourth Catalog of Interferometric Measurements of Binary Stars (Hartkopf et al. 2014, hereafter IC4).

Our "absolute" $\theta$ values are consistent with the values tabulated in WDS for all objects except for ADS 10849. For this object, $\Delta m_{V}=0.1$, the RTC file clearly shows that our quadrant determination of $\mathrm{Q}=2$ (South-East) is unquestionable (see Fig. 3). In IC4 there are 10 observations of this object, of which 3 were made by us, and the quadrant is always $\mathrm{Q}=4$. However, for our 3 observations the quadrant could not be determined so that we adopted the quadrant of the already published observations.

A possible explanation for this discrepancy may be that we observed with a $R$ filter, whereas most of the other observers used a $V$ filter. A different spectral type of the two components could account for a quadrant inversion.

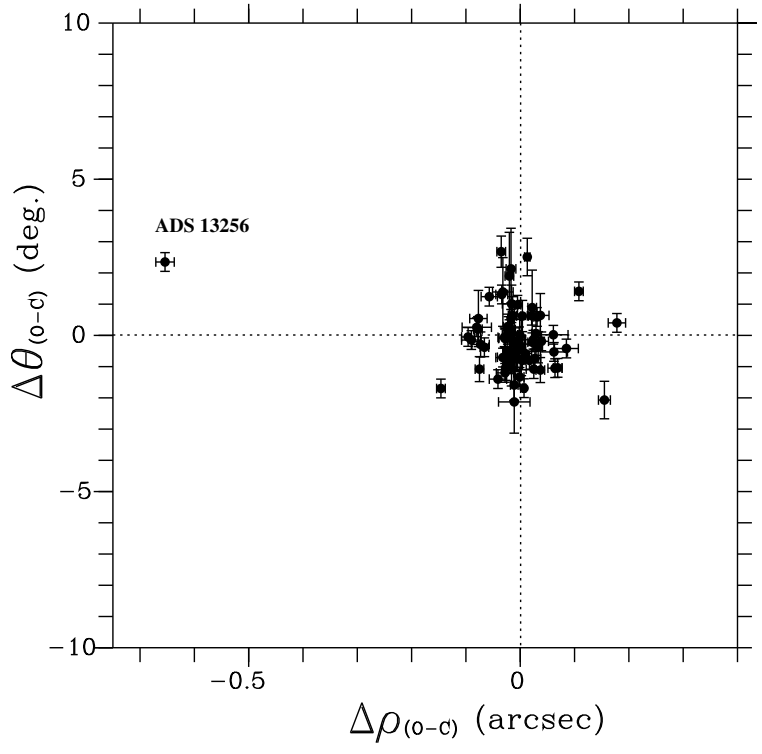

Fig. 4 Residuals of the measurements of Table 1 computed with the published orbits.

\subsection{Comparison with published ephemerides}

The $(O-C)$ (Observed minus Computed) residuals of the measurements for the systems with a known orbit in Table 1 are displayed in Cols. 13 and 14 for the separation $\rho$ and position angle $\theta$, respectively. Those residuals were obtained with a selection of valid orbits found in the OC6 catalog. We did not always use the most recent orbits since sometimes older orbits led to equivalent or even smaller residuals. For ADS 5871, 7203, 9378, 9578 and 11186, we also reported the residuals obtained with our revised orbit presented in Sect. 4 .

The residuals are plotted in Fig. 4. ADS 13256 which lies on the far left edge of this figure should be considered separately. It corresponds to the largest residual in Table $1\left(\Delta_{\rho}=-0^{\prime \prime} .65, \Delta \theta=2^{\circ} .3\right)$ with Hopmann (1973)'s orbit. This orbit was computed long ago, with insufficient data. The arc of the monitored orbit was very short in 1973, and has not yet increased sufficiently to enable a valid orbit calculation. Furthermore, the motion seems rectilinear, and it is likely that the two components of ADS 13256 are not gravitationally linked.

When excluding ADS 13256, Fig. 4 shows that the residuals have a rather small scatter. Indeed, the mean values computed with the residuals of Table 1 are $\left\langle\Delta \rho_{O-C}\right\rangle=-0^{\prime \prime} .005 \pm 0^{\prime \prime} .051$ and $\left\langle\Delta \theta_{O-C}\right\rangle=$ $-0^{\circ} .1 \pm 1^{\circ} .0$. The small values obtained for those offsets provide a good validation of our calibration (see Paper XII). 
Table 2 New orbital elements of ADS 1097, 5871, 7203, 7775, 9378, 9578 and 11186.

\begin{tabular}{|c|c|c|c|c|c|c|c|c|c|c|c|c|}
\hline$\overline{\mathrm{ADS}}$ & $\begin{array}{c}\Omega_{2000} \\
\left({ }^{\circ}\right)\end{array}$ & $\begin{array}{l}\omega \\
\left({ }^{\circ}\right)\end{array}$ & $\begin{array}{c}i \\
\left({ }^{\circ}\right)\end{array}$ & $e$ & $\begin{array}{c}T \\
(\mathrm{yr})\end{array}$ & $\begin{array}{c}P \\
(\mathrm{yr})\end{array}$ & $\begin{array}{c}n \\
\left({ }^{\circ} / \mathrm{yr}\right)\end{array}$ & $\begin{array}{c}a \\
\left({ }^{\prime \prime}\right)\end{array}$ & $\begin{array}{c}\mathrm{A} \\
\left({ }^{\prime \prime}\right)\end{array}$ & $\begin{array}{c}\mathrm{B} \\
\left({ }^{\prime \prime}\right)\end{array}$ & $\begin{array}{c}\mathrm{F} \\
\left({ }^{\prime \prime}\right)\end{array}$ & $\begin{array}{c}\mathrm{G} \\
\left({ }^{\prime \prime}\right)\end{array}$ \\
\hline 1097 & $\begin{array}{c}149.8 \\
-\end{array}$ & 242.8 & 141.0 & - & - & - & 1.237 & - & 7 & & -1 & 0 \\
\hline 5871 & $\begin{array}{l}38.5 \\
\pm 2.1\end{array}$ & $\begin{array}{c}259.5 \\
\pm 1.9\end{array}$ & $\begin{array}{l}144.7 \\
\pm 1.3\end{array}$ & $\begin{array}{c}0.933 \\
\pm 0.002\end{array}$ & $\begin{array}{c}1920.34 \\
\pm 0.01\end{array}$ & $\begin{array}{l}118.35 \\
\pm 0.46\end{array}$ & $\begin{array}{c}3.042 \\
\pm 0.012\end{array}$ & $\begin{array}{c}0.794 \\
\pm 0.020\end{array}$ & 8 & 0.40857 & 0.53747 & 0.57842 \\
\hline 7203 & $\begin{array}{c}102.1 \\
\pm 1.9\end{array}$ & $\begin{array}{c}332.4 \\
\pm 1.9\end{array}$ & $\begin{array}{c}145.4 \\
\pm 1.5\end{array}$ & & $\begin{array}{c}1917.39 \\
\pm 0.12\end{array}$ & $\begin{array}{c}970 \\
\pm 118\end{array}$ & $\begin{array}{l}0.3712 \\
\pm 0.005\end{array}$ & $\begin{array}{c}5.80 \\
\pm 0.14\end{array}$ & -3.24016 & 4.56214 & 3.57364 & 3.51430 \\
\hline 7775 & $\begin{array}{c}121.2 \\
-\end{array}$ & $\begin{array}{c}219.9 \\
-\end{array}$ & $\begin{array}{c}47.3 \\
-\end{array}$ & $\begin{array}{c}0.984 \\
-\end{array}$ & $\begin{array}{c}1966.983 \\
-\end{array}$ & $\begin{array}{c}139.789 \\
-\end{array}$ & $\begin{array}{c}2.575 \\
-\end{array}$ & $\begin{array}{c}0.470 \\
-\end{array}$ & 0.36167 & 1 & 8 & 0.38455 \\
\hline 9378 & $\begin{array}{l}71.4 \\
\pm 6.0\end{array}$ & $\begin{array}{c}171.0 \\
\pm 6.1\end{array}$ & $\begin{array}{l}164.5 \\
\pm 1.6\end{array}$ & $\begin{array}{l}0.5232 \\
\pm 0.002\end{array}$ & $\begin{array}{c}2060.738 \\
\pm 0.099\end{array}$ & $\begin{array}{l}88.933 \\
\pm 0.085\end{array}$ & $\begin{array}{c}4.048 \\
\pm 0.004\end{array}$ & $\begin{array}{c}0.327 \\
\pm 0.010\end{array}$ & 30 & 2183 & 29 & 0.05079 \\
\hline 9578 & $\begin{array}{l}63.79 \\
\pm 0.21\end{array}$ & $\begin{array}{l}51.26 \\
\pm 0.24\end{array}$ & $\begin{array}{l}58.56 \\
\pm 0.13\end{array}$ & $\begin{array}{c}0.6527 \\
\pm 0.0017\end{array}$ & $\begin{array}{c}1941.484 \\
\pm 0.024\end{array}$ & $\begin{array}{c}203.145 \\
\pm 1.3\end{array}$ & $\begin{array}{l}1.7721 \\
\pm 0.011\end{array}$ & $\begin{array}{c}1.21 \\
\pm 0.019\end{array}$ & -0.10724 & 0.89677 & -0.77119 & -0.67231 \\
\hline 186 & $\begin{array}{c}91.10 \\
-\end{array}$ & $\begin{array}{c}173.80 \\
-\end{array}$ & $\begin{array}{c}101.60 \\
-\end{array}$ & $\begin{array}{c}0.935 \\
-\end{array}$ & $\begin{array}{c}1890.086 \\
-\end{array}$ & $\begin{array}{c}344.837 \\
-\end{array}$ & $\begin{array}{c}1.04397 \\
-\end{array}$ & $\begin{array}{c}0.732 \\
-\end{array}$ & 86 & .72728 & -0.14478 & 0.08 \\
\hline
\end{tabular}

\section{Revised orbits of ADS 1097, 5871, 7203, 7775, 9378, 9578 and 11186}

In this section we present the new revised orbits that we have computed for ADS 1097, 5871, 7203, 7775, 9378, 9578 and 11186. Those objects belong to our list of regularly monitored couples with PISCO. The revision of those orbits was justified by the appearance of a systematic trend in the residuals of our last measurements and/or the existence of substantial number of new measurements since the computation of the last known orbit.

We have followed the same method for revising the orbits of those seven objects. Using our last measurements with PISCO and the other available observations contained in the data base maintained by the United States Naval Observatory (USNO), we first computed the preliminary orbital elements with the analytical method of Kowalsky (1873). We then used them as initial values for the least-squares method of Hellerich (1925). When convergence was achieved, Hellerich's method led to an improvement of the orbital elements (with the exception of the major axis) and to an estimation of the corresponding errors. The final value of the major axis was then set to the value that minimized the residuals in separation of Hellerich's solution.

The final orbital elements are presented in Table 2. The errors reported in this table were obtained by Hellerich's least-squares method. For ADS 1097, 7775, and 11186, the errors could not be estimated since Hellerich's method did not converge. The format of the tables contained in this section is self-explanatory, but a detailed description of those formats can be found in Papers VI and VII.

The $(O-C)$ residuals of the new orbits, restricted to the last observations for reasons of space, are given in Tables 3, 4, 5, 6, 7, 8, and 9, for ADS 1097, 5871, 7203,
$7775,9378,9578$ and 11186, respectively. The name of the observer is reported in the last column, using the US Naval Observatory convention.

The ephemerides for 2015-2025 are presented in Table 10. The apparent orbits are shown in Figs. 5 and 6 as solid lines. The observational data used for the calculation of the orbital elements are plotted as small crosses or, in the case of PISCO observations, as filled circles (that appear in red in the electronic version). The orientation of the graphs conforms to the convention adopted by the observers of visual binary stars. For each object, the location of the primary component is indicated with a big cross. The straight line going through this point is the line of apsides. An arrow shows the sense of rotation of the companion.

In Table 11, we present some physical parameters of those systems. The (total) visual magnitudes (Col. 3) and and the spectral types (Col. 5) were extracted from the SIMBAD data base. The difference of magnitude between the components (Col. 4) was taken from the IC4. The dynamical parallaxes (Col. 6) were derived from our orbital elements using Baize \& Romani (1946)'s method, with our revised formulae presented in Scardia et al. (2008b). In Col. 7, we report the Hipparcos parallaxes from ESA (1997) or the revised values from van Leeuwen (2007), as indicated in Col. 11. In Cols. 8, 9 and 10, we give the corresponding angular and linear sizes of the semi-major axis $a$ and the total mass $\mathfrak{M}_{\text {total }}$, respectively, that were computed from our orbital elements and the Hipparcos parallaxes.

\section{WDS 01213+1132 - BU 4 - ADS 1097 - HIP 6334}

This couple was discovered by S.W. Burnham, on October 24th 1872 in Chicago (Burnham, 1873). Burnham was using an outstanding 6-inch Clark refractor that he installed in the backyard of his house. This couple was rather well measured after this discovery, 

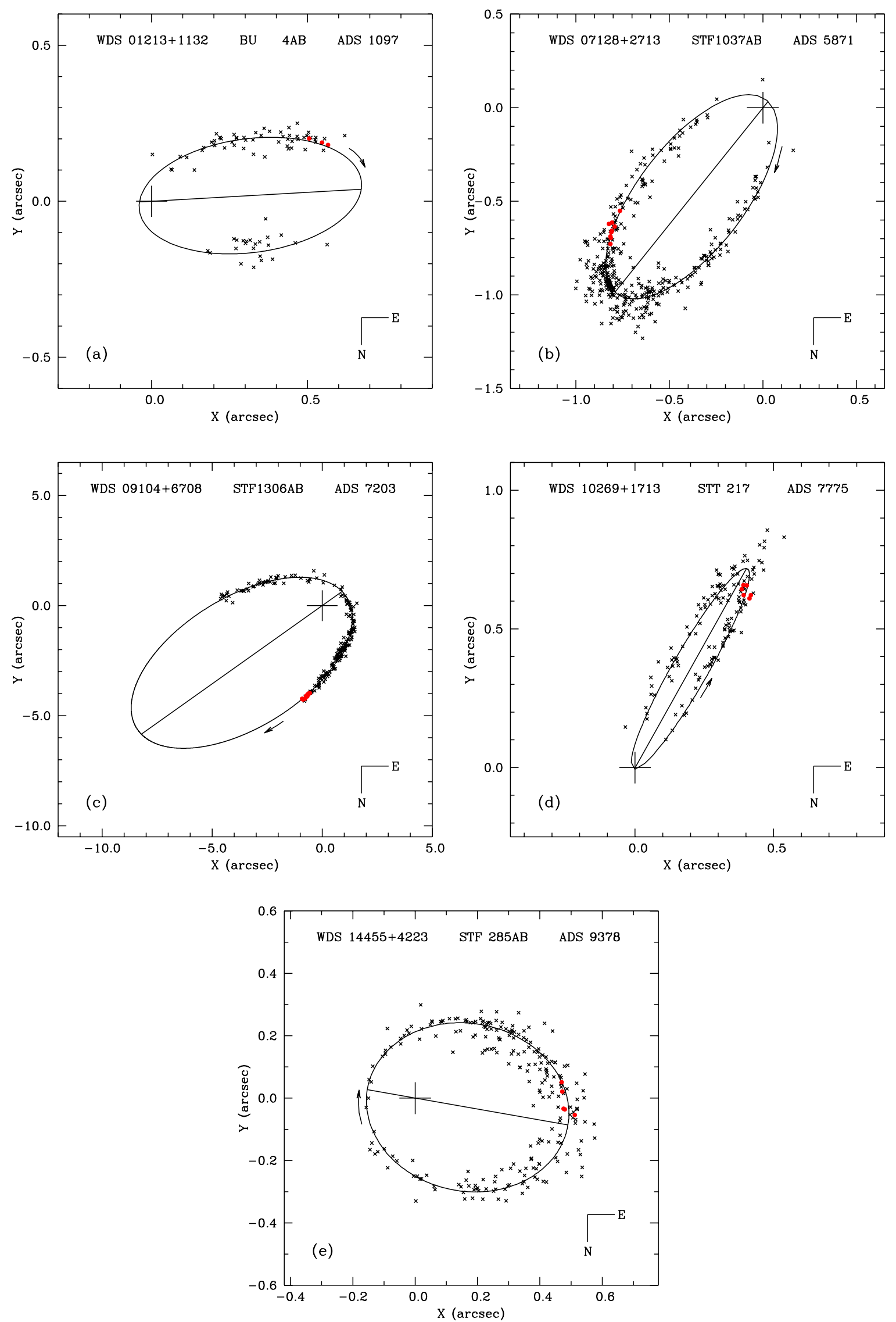

Fig. 5 New orbits of ADS 1097 (a), ADS 5871 (b), ADS 7203 (c), ADS 7775 (d), and ADS 9378 (e) The observations by PISCO are plotted as filled circles that appear in red in the electronic version of this paper. 

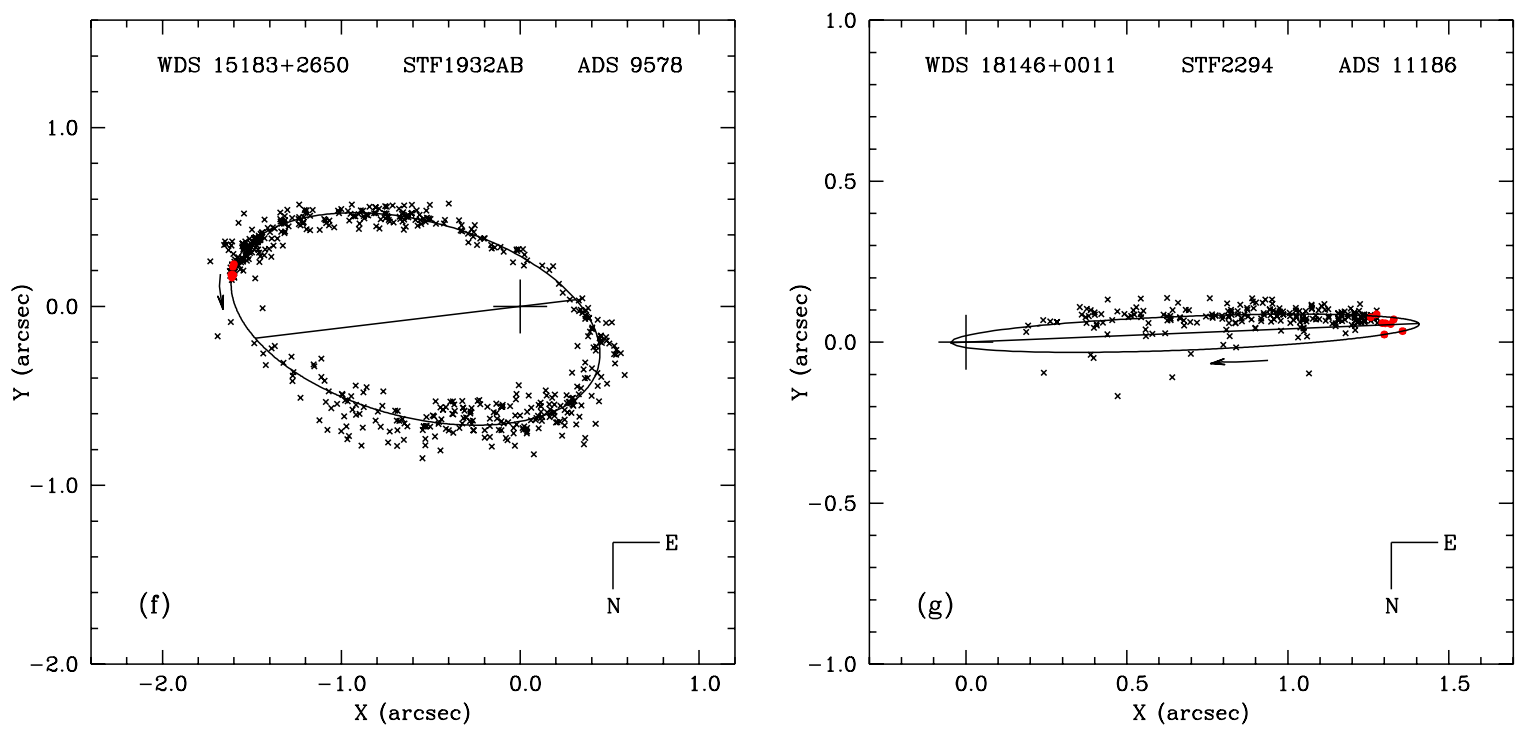

Fig. 6 New orbits of ADS 9578 (f) and ADS 11186 (g). The observations by PISCO are plotted as filled circles that appear in red in the electronic version of this paper.

Table 3 ADS 1097: O-C residuals of our new orbit (after 2001). The symbol ${ }^{P}$ indicates PISCO measurements.

\begin{tabular}{ccrl}
\hline Epoch & $\Delta \rho\left(\begin{array}{r}\mathrm{O}-\mathrm{C}) \\
\left({ }^{\prime \prime}\right)\end{array}\right.$ & $\begin{array}{r}\Delta \theta(\mathrm{O}-\mathrm{C}) \\
\left(^{\circ}\right)\end{array}$ & Observer \\
\hline 2001.873 & 0.032 & -4.2 & Hor \\
2001.873 & 0.022 & 0.5 & Hor \\
2005.900 & $0.020^{P}$ & $-0.6^{P}$ & Sca \\
2007.964 & $0.029^{P}$ & $-1.4^{P}$ & Sca \\
2007.995 & 0.089 & -0.3 & Gii \\
2008.885 & -0.068 & 9.3 & CTr \\
2008.888 & 0.022 & 1.0 & Orl \\
\hline
\end{tabular}

but it was unfortunately not observed between 1924 and 1945, which was the epoch when the periastron passage occurred.

The last orbit of ADS 1097 that we computed in Scardia et al. (2001) needed some revision because the residuals of the last measurements were beginning to show a systematic trend. Using the 91 measurements made since its discovery, we obtained the orbital elements reported in Table 2, with mean residuals of $\left\langle\Delta \rho_{O-C}\right\rangle=0^{\prime \prime} .042$ and $\left\langle\Delta \theta_{O-C}\right\rangle=3^{\circ} .6$. The orbit is rather eccentric with a lack of observations close to the periastron (see Fig. 5a). The parallax computed by van Leeuwen leads to an excessive mass, whereas the ESA parallax gives an acceptable result and is in very good agreement with the dynamical parallax.

\section{WDS 07128+2713 - STF 1037 - ADS 5871 - HIP 34860}

This couple was discovered by F.G.W. Struve in Dorpat (now Tartu, Estonia) during the binary star survey he made in 1825-1827 with the famous $25-\mathrm{cm}$
Table 4 ADS 5871: O-C residuals of our new orbit (after 2009). The symbol ${ }^{P}$ indicates PISCO measurements.

\begin{tabular}{ccrl}
\hline Epoch & $\begin{array}{r}\Delta \rho(\mathrm{O}-\mathrm{C}) \\
\left({ }^{\prime \prime}\right)\end{array}$ & $\begin{array}{r}\Delta \theta(\mathrm{O}-\mathrm{C}) \\
\left(^{\circ}\right)\end{array}$ & Observer \\
\hline 2009.139 & $-0.026^{P}$ & $-0.6^{P}$ & Sca \\
2009.153 & -0.020 & 0.6 & WSI \\
2009.201 & -0.020 & 0.2 & Ctt \\
2009.909 & -0.019 & 0.8 & FMR \\
2010.160 & 0.055 & -0.7 & StJ \\
2010.196 & $0.007^{P}$ & $-1.7^{P}$ & Pru \\
2011.178 & 0.042 & 0.8 & Thr \\
2012.156 & 0.028 & 0.7 & FMR \\
2012.156 & 0.028 & 0.7 & Bvd \\
2013.174 & $0.039^{P}$ & $0.3^{P}$ & Sca \\
2014.196 & $-0.013^{P}$ & $-0.6^{P}$ & Sca \\
\hline
\end{tabular}

Table 5 ADS 7203: O-C residuals of our new orbit (after 2009). The symbol ${ }^{P}$ indicates PISCO measurements.

\begin{tabular}{cccl}
\hline Epoch & $\begin{array}{r}\Delta \rho(\mathrm{O}-\mathrm{C}) \\
\left({ }^{\prime \prime}\right)\end{array}$ & $\begin{array}{r}\Delta \theta(\mathrm{O}-\mathrm{C}) \\
\left({ }^{\circ}\right)\end{array}$ & Observer \\
\hline 2009.207 & $0.016^{P}$ & $0.0^{P}$ & Sca \\
2009.359 & -0.166 & 1.9 & Ary \\
2010.196 & $0.113^{P}$ & $-0.0^{P}$ & Pru \\
2011.310 & -0.029 & 0.3 & Mwl \\
2011.313 & 0.111 & 0.7 & Mwl \\
2012.203 & $0.094^{P}$ & $-0.4^{P}$ & Pru \\
2012.217 & 0.162 & 0.7 & Mwl \\
2012.251 & 0.036 & 0.1 & Dru \\
2012.284 & 0.059 & -0.4 & Dal \\
2013.287 & $0.056^{P}$ & $-0.1^{P}$ & Sca \\
2014.196 & $0.016^{P}$ & $-0.3^{P}$ & Sca \\
\hline
\end{tabular}


Table 10 New ephemerides of ADS 1097, 5871, 7203, 7775, 9378, 9578 and 11186.

\begin{tabular}{|c|c|c|c|c|c|c|c|c|c|c|c|c|c|c|}
\hline \multirow[b]{2}{*}{ Epoch } & \multicolumn{2}{|c|}{ ADS 1097} & \multicolumn{2}{|c|}{ ADS 5871} & \multicolumn{2}{|c|}{ ADS 7203} & \multicolumn{2}{|c|}{ ADS 7775} & \multicolumn{2}{|c|}{ ADS 9378} & \multicolumn{2}{|c|}{ ADS 9578} & \multicolumn{2}{|c|}{ ADS 11186} \\
\hline & $\begin{array}{c}\rho \\
\left(^{\prime \prime}\right)\end{array}$ & $\begin{array}{c}\theta \\
\left(^{\circ}\right)\end{array}$ & $\begin{array}{c}\rho \\
\left(^{\prime \prime}\right)\end{array}$ & $\begin{array}{c}\theta \\
\left(^{\circ}\right)\end{array}$ & $\begin{array}{c}\rho \\
\left(^{\prime \prime}\right)\end{array}$ & $\begin{array}{c}\theta \\
\left(^{\circ}\right)\end{array}$ & $\begin{array}{c}\rho \\
\left(^{\prime \prime}\right)\end{array}$ & $\begin{array}{c}\theta \\
\left(^{\circ}\right)\end{array}$ & $\begin{array}{c}\rho \\
\left({ }^{\prime \prime}\right)\end{array}$ & $\begin{array}{c}\theta \\
\left(^{\circ}\right)\end{array}$ & $\begin{array}{c}\rho \\
\left({ }^{\prime \prime}\right)\end{array}$ & $\begin{array}{c}\theta \\
\left(^{\circ}\right)\end{array}$ & $\begin{array}{c}\rho \\
\left({ }^{\prime \prime}\right)\end{array}$ & $\begin{array}{c}\theta \\
\left(^{\circ}\right)\end{array}$ \\
\hline 2015.0 & 0.588 & 107.4 & 0.940 & 305.9 & 4.337 & 347.9 & 0.783 & 148.5 & 0.497 & 81.9 & 1.622 & 265.2 & 1.319 & 93.4 \\
\hline 2016.0 & 0.591 & 107.1 & 0.920 & 305.3 & 4.369 & 347.6 & 0.787 & 148.6 & 0.498 & 80.5 & 1.621 & 265.6 & 1.323 & 93.4 \\
\hline 2017.0 & 0.594 & 106.9 & 0.900 & 304.6 & 4.400 & 347.3 & 0.791 & 148.7 & 0.498 & 79.0 & 1.620 & 266.0 & 1.330 & 93.3 \\
\hline 2018.0 & 0.597 & 106.7 & 0.879 & 303.9 & 4.432 & 346.9 & 0.795 & 148.8 & 0.497 & 77.6 & 1.619 & 266.4 & 1.333 & 93.3 \\
\hline 2019.0 & 0.600 & 106.5 & 0.857 & 303.1 & 4.463 & 346.6 & 0.798 & 148.9 & 0.497 & 76.2 & 1.617 & 266.8 & 1.336 & 93.3 \\
\hline 2020.0 & 0.602 & 106.2 & 0.834 & 302.3 & 4.494 & 346.3 & 0.802 & 149.0 & 0.495 & 74.7 & 1.615 & 267.2 & 1.339 & 93.2 \\
\hline 2021.0 & 0.605 & 106.0 & 0.810 & 301.5 & 4.525 & 346.0 & 0.805 & 149.1 & 0.494 & 73.3 & 1.613 & 267.6 & 1.342 & 93.2 \\
\hline 2022.0 & 0.608 & 105.8 & 0.785 & 300.6 & 4.556 & 345.7 & 0.807 & 149.2 & 0.492 & 71.8 & 1.610 & 268.0 & 1.345 & 93.2 \\
\hline 2023.0 & 0.610 & 105.6 & 0.759 & 299.7 & 4.587 & 345.4 & 0.810 & 149.3 & 0.490 & 70.3 & 1.608 & 268.3 & 1.348 & 93.2 \\
\hline 2024.0 & 0.613 & 105.3 & 0.732 & 298.6 & 4.617 & 345.1 & 0.812 & 149.4 & 0.487 & 68.8 & 1.604 & 268.7 & 1.351 & 93.1 \\
\hline 2025.0 & 0.615 & 105.1 & 0.704 & 297.5 & 4.648 & 344.8 & 0.814 & 149.5 & 0.484 & 67.3 & 1.601 & 269.1 & 1.353 & 93.1 \\
\hline
\end{tabular}

Table 6 ADS 7775: O-C residuals of our new orbit (after 2007). The symbol ${ }^{P}$ indicates PISCO measurements.

\begin{tabular}{cccl}
\hline Epoch & $\begin{array}{r}\Delta \rho(\mathrm{O}-\mathrm{C}) \\
\left({ }^{\prime \prime}\right)\end{array}$ & $\begin{array}{r}\Delta \theta(\mathrm{O}-\mathrm{C}) \\
\left({ }^{\circ}\right)\end{array}$ & Observer \\
\hline 2007.261 & $-0.006^{P}$ & $-1.7^{P}$ & Pru \\
2008.251 & -0.009 & 1.3 & $\mathrm{Gii}$ \\
2008.284 & 0.006 & 0.7 & $\mathrm{Gii}$ \\
2008.284 & 0.023 & 0.8 & $\mathrm{Gii}$ \\
2008.314 & $-0.000^{P}$ & $-1.6^{P}$ & Pru \\
2009.301 & 0.025 & $1.9^{P}$ & Ant \\
2009.330 & $-0.006^{P}$ & $1.3^{P}$ & Sca \\
2010.188 & -0.004 & 1.0 & Los \\
2012.380 & $-0.006^{P}$ & $1.1^{P}$ & Sca \\
\hline
\end{tabular}

Table 7 ADS 9378: O-C residuals of our new orbit (after 2005). The symbol ${ }^{P}$ indicates PISCO measurements.

\begin{tabular}{ccrl}
\hline Epoch & $\begin{array}{r}\Delta \rho(\mathrm{O}-\mathrm{C}) \\
\left({ }^{\prime \prime}\right)\end{array}$ & $\begin{array}{r}\Delta \theta(\mathrm{O}-\mathrm{C}) \\
\left({ }^{\circ}\right)\end{array}$ & Observer \\
\hline 2005.404 & $0.000^{P}$ & $-0.0^{P}$ & Sca \\
2006.320 & -0.007 & -0.5 & WSI \\
2007.312 & 0.007 & 0.3 & Hrt \\
2007.380 & -0.011 & 2.6 & $\mathrm{Alz}$ \\
2007.381 & -0.005 & -1.3 & $\mathrm{Gii}$ \\
2007.542 & $-0.010^{P}$ & $-0.4^{P}$ & $\mathrm{Sca}$ \\
2010.223 & 0.052 & $1.3^{P}$ & Los \\
2011.485 & $-0.015^{P}$ & $-1.1^{P}$ & Sca \\
2013.448 & $-0.015^{P}$ & $1.5^{P}$ & Sca \\
2013.462 & $0.019^{P}$ & $-0.2^{P}$ & Sca \\
\hline
\end{tabular}

refractor built by Fraunhofer. The first measurement was made in March 1827 (Struve, 1837).

Both the orbit of Scardia (1983, last obs. 1980.09) and the most recent one computed by Söderhjelm (1999) begin to show systematic residuals both in $\rho$ and $\theta$. Consequently we took advantage of the 130 new measurements obtained since 1980 and revised our first orbit. The new orbital elements are displayed in Table 2 and the apparent orbit is plotted in Fig. 5b. They
Table 8 ADS 9578: O-C residuals of our new orbit (after 2009). The symbol ${ }^{P}$ indicates PISCO measurements.

\begin{tabular}{crrl}
\hline Epoch & $\begin{array}{r}\Delta \rho(\mathrm{O}-\mathrm{C}) \\
\left({ }^{\prime \prime}\right)\end{array}$ & $\begin{array}{r}\Delta \theta(\mathrm{O}-\mathrm{C}) \\
\left(^{\circ}\right)\end{array}$ & Observer \\
\hline 2009.487 & 0.000 & 1.7 & Nvl \\
2009.649 & -0.010 & -0.3 & WSI \\
2010.388 & 0.014 & -0.4 & Los \\
2010.435 & -0.131 & 0.5 & WSI \\
2010.456 & 0.009 & 0.5 & Ant \\
2010.538 & $-0.007^{P}$ & $0.3^{P}$ & Pru \\
2011.444 & -0.012 & 0.1 & Bvd \\
2012.457 & $0.003^{P}$ & $-0.5^{P}$ & Sca \\
2013.307 & -0.017 & -0.3 & RAO \\
2013.448 & $-0.003^{P}$ & $-0.4^{P}$ & Sca \\
\hline
\end{tabular}

Table 9 ADS 11186: O-C residuals of our new orbit (after 2008). The symbol ${ }^{P}$ indicates PISCO measurements.

\begin{tabular}{cccl}
\hline Epoch & $\begin{array}{r}\Delta \rho(\mathrm{O}-\mathrm{C}) \\
\left({ }^{\prime \prime}\right)\end{array}$ & $\begin{array}{rll}\Delta \theta(\mathrm{O}-\mathrm{C}) \\
\left({ }^{\circ}\right)\end{array}$ & Observer \\
\hline 2008.665 & $0.021^{P}$ & $-1.0^{P}$ & Pru \\
2009.266 & 0.029 & -0.4 & Tok \\
2009.440 & 0.001 & -0.9 & FMR \\
2009.608 & -0.004 & -2.4 & WSI \\
2009.686 & $0.003^{P}$ & $-0.2^{P}$ & Sca \\
2010.500 & 0.050 & -2.0 & Los \\
2011.660 & $0.023^{P}$ & $-0.8^{P}$ & Sca \\
2012.635 & $0.034^{P}$ & $-0.7^{P}$ & Pru \\
2013.675 & $0.022^{P}$ & $-0.9^{P}$ & Sca \\
\hline
\end{tabular}

lead to mean residuals of $0^{\prime \prime} .073$ and $1^{\circ} .6$, for $\rho$ and $\theta$, respectively. The uncertainties of the orbital elements are very small and this orbit is close to being definitive. Indeed, the measurements are numerous (426 observations have been made since its discovery in 1827) and nearly two orbital revolutions have been well monitored by the observations. However the orbit is very eccentric $(e=0.933)$ and the last periastron passage could not 
Table 11 Physical parameters $\left(\pi_{\text {dyn }}, a\right.$ and $\left.\mathfrak{M}_{\text {total }}\right)$ derived from the new orbital elements.

\begin{tabular}{|c|c|c|c|c|c|c|c|c|c|c|}
\hline $\mathrm{ADS}$ & HIP & $m_{V}$ & $\Delta m_{V}$ & Spectral type & $\begin{array}{l}\pi_{\text {dyn }} \\
(\text { mas })\end{array}$ & $\begin{array}{l}\pi_{\mathrm{HIP}} \\
(\mathrm{mas})\end{array}$ & $\left({ }^{\prime \prime}\right)$ & (AU) & $\begin{array}{r}\mathfrak{M}_{\text {total }} \\
\left(\mathrm{M}_{\odot}\right) \\
\end{array}$ & Source of $\pi_{\mathrm{HIP}}$ \\
\hline 1097 & 6334 & 6.90 & 1.3 & F0V & 6.2 & $\begin{array}{c}6.16 \\
\pm 1.41\end{array}$ & 0.430 & $\begin{aligned} & 70 \\
\pm & 16^{(1)}\end{aligned}$ & $\begin{aligned} & 4.0 \\
\pm & 2.8^{(1)}\end{aligned}$ & ESA (1997) \\
\hline$"$ & $"$ & $"$ & $"$ & $"$ & $"$ & $\begin{array}{c}4.84 \\
\pm 0.64\end{array}$ & $"$ & $\begin{aligned} & 89 \\
\pm & 12^{(1)}\end{aligned}$ & $\begin{aligned} & 8.3 \\
\pm & 3.3^{(1)}\end{aligned}$ & van Leeuwen (2007) \\
\hline 5871 & 34860 & 6.44 & 0.0 & $\mathrm{~F} 8 \mathrm{~V}$ & 25.2 & $\begin{array}{l}22.65 \\
\pm 2.03\end{array}$ & $\begin{array}{r}0.794 \\
\pm 0.020\end{array}$ & $\begin{array}{r}35 \\
\pm 3\end{array}$ & $\begin{array}{r}3.1 \\
\pm 0.9\end{array}$ & ESA (1997) \\
\hline$"$ & $"$ & $"$ & $"$ & $"$ & $"$ & $\begin{array}{l}23.54 \\
\pm 0.55\end{array}$ & $"$ & $\begin{array}{r}34 \\
\pm 1\end{array}$ & $\begin{array}{r}2.7 \\
\pm 0.3\end{array}$ & van Leeuwen (2007) \\
\hline 7203 & 45038 & 4.80 & 4.0 & $\mathrm{~F} 7 \mathrm{~V}+\mathrm{K} 2 \mathrm{~V}$ & 47.3 & $\begin{array}{l}48.87 \\
\pm 0.62\end{array}$ & $\begin{array}{r}5.80 \\
\pm 0.14\end{array}$ & $\begin{array}{r}119 \\
\pm 3\end{array}$ & $\begin{array}{r}1.8 \\
\pm 0.5\end{array}$ & ESA (1997) \\
\hline$"$ & $"$ & $"$ & $"$ & $"$ & $"$ & $\begin{array}{l}49.07 \\
\pm 0.37\end{array}$ & $"$ & $\begin{array}{r}118 \\
\pm 3\end{array}$ & $\begin{array}{r}1.8 \\
\pm 0.5\end{array}$ & van Leeuwen (2007) \\
\hline 7775 & 51145 & 7.43 & 0.5 & $\mathrm{~F} 8 \mathrm{~V}$ & 12.8 & $\begin{array}{l}11.67 \\
\pm 2.70\end{array}$ & 0.47 & $\begin{array}{l}40 \\
\pm 9^{(1)}\end{array}$ & $\begin{aligned} & 3.3 \\
\pm & 2.3^{(1)}\end{aligned}$ & ESA (1997) \\
\hline$"$ & $"$ & $"$ & $"$ & $"$ & $"$ & $\begin{array}{l}11.80 \\
\pm 1.44\end{array}$ & $"$ & $\begin{array}{l}40 \\
\pm 5^{(1)}\end{array}$ & $\begin{array}{c}3.2 \\
\pm \\
1.2^{(1)}\end{array}$ & 1) van Leeuwen (2007) \\
\hline 9378 & 72153 & 7.33 & 0.1 & F5V & 11.9 & $\begin{array}{l}11.81 \\
\pm 1.26\end{array}$ & $\begin{array}{r}0.327 \\
\pm 0.010\end{array}$ & $\begin{array}{r}28 \\
\pm 3\end{array}$ & $\begin{array}{r}2.7 \\
\pm 0.9\end{array}$ & ESA (1997) \\
\hline$"$ & $"$ & $"$ & $"$ & $"$ & $"$ & $\begin{array}{l}11.93 \\
\pm 0.77\end{array}$ & $"$ & $\begin{array}{l}27 \\
\pm 2\end{array}$ & $\begin{array}{r}2.6 \\
\pm 0.6\end{array}$ & van Leeuwen (2007) \\
\hline 9578 & 74893 & 6.58 & 0.1 & G0V & 27.1 & $\begin{array}{l}26.10 \\
\pm 1.27\end{array}$ & $\begin{array}{r}1.21 \\
\pm 0.019\end{array}$ & $\begin{array}{l}46 \\
\pm 2\end{array}$ & $\begin{array}{r}2.4 \\
\pm 0.4\end{array}$ & ESA (1997) \\
\hline$"$ & $"$ & $"$ & $"$ & $"$ & $"$ & $\begin{array}{l}27.79 \\
\pm 0.81\end{array}$ & $"$ & $\begin{array}{l}44 \\
\pm 1\end{array}$ & $\begin{array}{r}2.0 \\
\pm 0.2\end{array}$ & van Leeuwen (2007) \\
\hline 11186 & 89393 & 7.67 & 0.3 & F5V & 10.8 & $\begin{array}{l}11.45 \\
\pm 1.97\end{array}$ & 0.732 & $\begin{array}{c}64 \\
\pm 11^{(1)}\end{array}$ & $\begin{array}{c}2.2 \\
\pm 1.1^{(1)}\end{array}$ & ESA (1997) \\
\hline$"$ & $"$ & $"$ & $"$ & $"$ & $"$ & $\begin{array}{c}8.74 \\
\pm 1.54\end{array}$ & $"$ & $\begin{aligned} & 84 \\
\pm & 15^{(1)}\end{aligned}$ & $\begin{aligned} & 4.9 \\
\pm & 2.6^{(1)}\end{aligned}$ & van Leeuwen (2007) \\
\hline
\end{tabular}

(1) lower estimate of the error, using the parallax error only, and neglecting all the other (unknown) errors.

monitored due to the small angular separation. Indeed, at periastron $\rho=(1-e) a \approx 0^{\prime \prime} .05$. The next periastron passage in the years 2036-2040 should enable our successors to definitely precise this orbit.

The companion has nearly the same luminosity as the primary $\left(\Delta m_{V}=0.03\right)$ and we can therefore assume that this system is made of two F8V stars. The dynamical parallax is in good agreement with the Hipparcos measurement and the total mass is consistent with the theoretical value (both for ESA, 1997, and van Leeuwen, 2007).

\section{WDS 09104+6708 - STF 1306 - ADS 7203 - HIP $45038-\sigma_{2}$ UMa}

This couple was discovered by W. Herschel on 1782 June 2nd and classified as H III 54, whereas it was numbered \#1306 in F.G.W. Struve's catalog (Struve, 1827). More historical information can be found in Scardia (1980). This couple has a long period orbit with a high eccentricity. The observations are numerous but only cover an arc of orbit straddling the periastron passage of 1917 (see Fig. 5c). The previous orbit of Scardia (1985, last obs. 1985.27) was computed long ago and needed some revision to take ad- vantage of the 38 new measurements made since 1985 . Among those, 14 were obtained with PISCO. This couple was particularly difficult to measure because of the large magnitude difference between the two components: $\Delta m_{V} \approx 4$, which is close to the limit of our capabilities with the current detector of PISCO (see Fig. 1c).

A total of 223 observations have been made since its discovery in 1782 . We also used an unpublished micrometer observation made by MS with the $23 \mathrm{~cm}-$ finder of the Ruths telescope in Merate:

$$
1993.192356^{\circ} .43^{\prime \prime} .660 .232 \text { Sca Ma }
$$

(in the WDS format). The orbital elements reported in Table 2 fit the observations quite well (see Fig. 5c), with mean residuals of $\left\langle\Delta \rho_{O-C}\right\rangle=0.114^{\prime \prime}$ and $\left\langle\Delta \theta_{O-C}\right\rangle=$ $1.85^{\circ}$. Only part of the orbit has been monitored yet, which explains the large uncertainty of the period. However, as the dispersion of the measures is quite small, the other orbital elements are rather well determined with a small uncertainty.

The value derived from our orbital elements and the Hipparcos parallax is in good agreement with the theoretical value expected for a system F7V+K2V (see 
Table 11). The dynamical parallax computed with our formula (Scardia et al., 2008b) is also consistent with the Hipparcos parallax.

\section{WDS 10269+1713 - STT 217 - ADS 7775 - HIP 51145}

This couple was discovered by O Struve (1843) with the 38-cm Merz-Mahler Poulkova refractor, during his survey aiming at founding new double stars (August 1841 - December 1842). It has been regularly observed since its discovery, except during the period 1960-1970, when the angular separation was very small. Two orbits have been computed by Heintz (1963 and 1975).

ADS 7775 is also a couple that we regularly observe with PISCO (6 observations since 2004). The last orbit of Heintz (1975) is still acceptable but begins to show a systematic trend in the residuals. The most striking feature of the orbit of ADS 7775 is a very high eccentricity (one of the most highly eccentric orbits amongst the known visual binaries). The apparent orbit is also very eccentric with a lack of observations close to the periastron (see Fig. 5d). Indeed the angular separation at the periastron passage (1966.983) was very small (less than $0^{\prime \prime} .1$ ) which made the measurements impossible with the available instrumentation at that epoch.

From the 159 available measurements obtained since its discovery, we obtained the orbital elements reported in Table 2 that lead to mean residuals of $\left\langle\Delta \rho_{O-C}\right\rangle=0.073^{\prime \prime}$ and $\left\langle\Delta \theta_{O-C}\right\rangle=1.94^{\circ}$. Leastsquares convergence could not be obtained, so we cannot give the uncertainties in this table. Clearly measurements close to the periastron are needed to constrain this orbit. But unfortunately, with a period estimated at $140 \mathrm{yr}$, the next periastron passage should occur in 2107 ..

The companion has a luminosity close to that of the primary $\left(\Delta m_{V}=0.5\right)$. We can therefore assume that this system is made of two main sequence F-type stars (see Table 11). The dynamical parallax is in good agreement with the Hipparcos measurement and the total mass is consistent with the expected theoretical value (both for ESA, 1997, and van Leeuwen, 2007).

\section{WDS 14455+4223 - STT 285 - ADS 9378 - HIP 72153}

Like STT 217, the couple STT 285 was also discovered by O Struve (1843) with the 38-cm Merz-Mahler Poulkova refractor, during his double star survey of 1841-1842. It has been regularly observed since its discovery, and the measurements are numerous. It should be noted, that in spite of the significant luminosity difference between the two components $\left(\Delta m_{V} \approx 0.8\right)$, quadrant reversals are frequent in the position angle measurements reported by the observers. This is surprisingly also true for some recent observations with big telescopes.

Since the first orbit computed by Burnham (1891), many others have been published for this couple. The previous orbit Couteau (1973) was still acceptable but needed some revision to profit from the 75 new accurate measurements obtained since 1973. Our new orbit of ADS 9378 was calculated by fitting the whole set of 282 observations, made since its discovery. The orbital elements reported in Table 2 fit the observations well (see Fig. 5e), with mean residuals of $0^{\prime \prime} .040$ and $2^{\circ} .3$ for $\rho$ and $\theta$, respectively. In the total list of measurements, we had to remove 28 aberrant measurements leading to residuals larger than $6 .^{\circ}$ and $0^{\prime \prime} .11$.

The companion has a luminosity close to that of the primary $\left(\Delta m_{V}=0.1\right)$. We can therefore assume that this system is made of two F5V stars (see Table 11). The dynamical parallax is in good agreement with the Hipparcos measurement and the total mass is in agreement with the expected theoretical value (both for ESA, 1997, and van Leeuwen, 2007).

\section{WDS 15183+2650 - STF 1932 - ADS 9578 - HIP 74893}

Like STF 1037, this couple was discovered by F.G.W. Struve in Dorpat during the binary star survey he made in 1825-1827 with the Fraunhofer 25 -cm refractor (Struve, 1827). ADS 9578 was first measured in July 1828 (Struve, 1837) and has been then regularly observed. The measurements are numerous. The companion has nearly completed a full revolution around the primary star since its discovery.

The last orbits computed by Heintz (1965) and Söderhjelm (1999) are still acceptable but both begin to show a systematic trend in the residuals. Taking advantage of the 41 new measurements obtained since 2000, we computed a new orbit from a total list of 576 measurements (see Fig. 6f). After rejecting 41 aberrant observations having residuals larger than 3.6 degrees in $\theta$ and 0.25 arcseconds in $\rho$, we obtained the final orbital elements reported in Table 2 . The corresponding orbit has mean residuals of $0^{\prime \prime} .077$ and $1^{\circ} .4$ for $\rho$ and $\theta$, respectively. The computed systemic mass using Hipparcos parallax (see Table 11) is in good agreement with theoretical values. The orbital elements have small uncertainties (see Table 2) and this orbit can be considered as definitive.

The companion has a luminosity close to that of the primary $\left(\Delta m_{V}=0.1\right)$. We can therefore assume that this system is made of two G0V stars. The values found for $\mathfrak{M}_{\text {total }}$ are compatible with the theoretical values (see Table 11).

\section{WDS 18146+0011 - STF 2294 - ADS 11186 - HIP 89393}

Like STF 1037 and STF 1932, this couple was discovered by F.G.W. Struve in Dorpat during his 18251827 binary star survey with the Fraunhofer 25-cm refractor. It was first measured in July 1828 (Struve, 1837). This couple was regularly observed in the XXth century, but the measurements made in the XIXth century are scarce and scattered. The apparent orbit of 
ADS 11186 has a high eccentricity with a lack of measurements close to the periastron (see Fig. 6g). Only three orbits have been computed until now, by Wilson $(1933,1935)$ and Luyten (1934). Taking advantage of the eighty years of measurements obtained since that epoch, we computed new orbital elements for this couple. From the 238 available observations made since its discovery in 1831, we obtained the orbital elements displayed in Table 2. They lead to mean residuals of $\left\langle\Delta \rho_{O-C}\right\rangle=0^{\prime \prime} .078$ and $\left\langle\Delta \theta_{O-C}\right\rangle=2^{\circ} .0$. Errors could not estimated since convergence was not reached with Hellerich's least-squares method. The representation of the observations by our orbital elements is good (especially in the plots $\rho$ or $\theta$ versus time which are more appropriate to estimate the validity of our solution than the plot of Fig. $6 \mathrm{~g}$ since the eccentricity of this orbit is very high). The parallax computed by van Leeuwen leads to excessive masses, but the ESA parallax gives acceptable results and is in good agreement with the dynamical parallax.

\section{Conclusion}

In 2013, we obtained 134 new measurements of 129 visual binaries with PISCO in Merate, with an average accuracy of $0^{\prime \prime} .01$ for the angular separation and $0^{\circ} .5$ for the position angles.

We also presented new orbital elements computed for ADS 1097, 5871, 7203, 7775, 9378, 9578 and 11186 Those orbits were partly derived from PISCO observations. The total mass values we have obtained are compatible with the expected theoretical values. The dynamic parallaxes computed with our revised formulae presented in Scardia et al. (2008b) are in very good agreement with Hipparcos values.

The total number of measurements made with PISCO in Merate since its installation in 2004 now exceeds 3300. Our group has thus provided a good contribution to the continuing monitoring of long period visual binary systems, which is important for refining systemic stellar masses.

Acknowledgements. We are very grateful to Daniel Gaffé (Laboratoire d'Electronique Antennes et Télécommunication Nice, France) for kindly repairing our ICCD camera in July 2013, and thus allowing us to continue our observing programme with PISCO. We thank the members of the United States Naval Observatory, Washington DC, for kindly sending on request some lists of measurements of visual binaries. This work has made use of the Washington Double Star Catalog (http://ad.usno.navy.mil/wds/wds), the "Fourth Catalog of Interferometric Measurements of Binary Stars" (http://ad.usno. navy.mil/wds/int4), and the "Sixth Catalog of Orbits of Visual Binary Stars" (http://ad.usno.navy.mil/wds/orb6), maintained at the U.S. Naval Observatory. We also used the SIMBAD astronomical data base (http://simbad.u-strasbg.fr/simbad) operated by the Centre de Données Astronomiques de Strasbourg (France).

\section{References}

Aitken, R.G., 1932, "New General Catalogue of Double Stars", Carnegie Institute, Washington

Aristidi, E., Carbillet, M., Lyon, J.-F., Aime, C., 1997, A\&AS, 125, 139

Baize, P., Romani, L., 1946, Ann. Astrophys. 9, 13

Burnham, S.W., 1873, MNRAS 33, 351

Burnham, S.W., 1891, Sidereal Messenger 10, 273

Couteau, P., 1973, A\&AS 12, 127

ESA: 1997, The Hipparcos and Tycho Catalogues, ESA SP1200, ESA Publications Division, Noordwijk

Hartkopf, W.I., Mason, B.D., 2014, "Sixth Catalog of Orbits of Visual Binary Stars" http://ad.usno.navy.mil/wds/orb6.html (OC6)

Hartkopf, W.I., Mason, B.D., Wycoff, G.L., McAlister, H.A., 2014, "Fourth Catalog of Interferometric Measurements of Binary Stars" http://ad.usno.navy.mil/wds/int4.html (IC4)

Heintz, W.D., 1963, Veroff. Sternw. München 5, 247

Heintz, W.D., 1965, Veroff. Sternw. München 7, 7

Heintz, W.D., 1975, ApJS, 29, 331

Hellerich, J., 1925, Astron. Nach. 223, 335

Hopmann, J, 1973, Astron. Mitt. Wien, 13, 322

Kowalsky, M., 1873, Procès-verbaux de l'Université Impériale de Kasan

Luyten, W.J., 1934, AJ, 43, 105

Mason, B.D., Wycoff, G.L., Hartkopf, W.I., 2014, "Washington Double Star Catalog" http://ad.usno.navy.mil/wds/wds.html (WDS)

Prieur, J.-L, Koechlin, L., André, C., Gallou, G., Lucuix, C., 1998, Experimental Astronomy, vol 8, Issue 4, 297

Prieur, J.-L., Scardia, M., Pansecchi, L., Argyle, R.W., Sala, M., Ghigo, M., Koechlin, L., Aristidi, E., 2008, MNRAS, 387, 772 (Paper V)

Prieur, J.-L., Scardia, M., Pansecchi, L., Argyle, R.W., Sala, M., 2009, MNRAS, 395, 907 (Paper VII)

Prieur, J.-L., Scardia, M., Pansecchi, L., Argyle, R.W., Sala, M., 2010, MNRAS, 407, 1913 (Paper IX)

Prieur, J.-L., Scardia, M., Pansecchi, L., Argyle, R.W., Sala, M., 2012, MNRAS, 422, 1057 (Paper XI)

Prieur, J.-L., Scardia, M., Pansecchi, L., Argyle, R.W., Zanutta, A., Aristidi, E., 2014, Astron. Nach., 335, 817 (Paper XIII)

Scardia, M., 1980, Astron. Nach., 301, 95

Scardia, M., 1983, Astron. Nach., 304, 29

Scardia, M., 1985, IAU Commission 26, Inf. Circ. n. 95

Scardia, M., Prieur, J.-L., Koechlin L., Aristidi, E., 2001, Astron. Nach., 322, 161

Scardia, M., Prieur, J.-L., Sala, M., Ghigo, M., Koechlin, L., Aristidi, E., Mazzoleni, F., 2005, MNRAS, 357, 1255 (with erratum in MNRAS 362, 1120) (Paper I)

Scardia, M., Prieur, J.-L., Pansecchi, L., Argyle, R.W., Sala, M., Ghigo, M., Koechlin, L., Aristidi, E., 2006, MNRAS, 367, 1170 (Paper II)

Scardia, M., Prieur, J.-L., Pansecchi, L., Argyle, R.W., Basso, S., Sala, M., Ghigo, M., Koechlin, L., Aristidi, E., 2007, MNRAS, 374, 965 (Paper III)

Scardia, M., Prieur, J.-L., Pansecchi, L., Argyle, R.W., Sala, M., Basso, S., Ghigo, M., Koechlin, L., Aristidi, E., 2008a, Astron. Nach., 329, 1, 54 (Paper IV)

Scardia, M., Prieur, J.-L., Pansecchi, L., Argyle, R.W., 2008b, Astron. Nach., 329, 379 
Scardia M., Prieur J.-L., Pansecchi L., Argyle R.W., Sala M., 2009, Astron. Nach., 330, 1, 55 (Paper VI)

Scardia, M., Prieur, J.-L., Pansecchi, L., Argyle, R.W., Sala, M., 2010, Astron. Nach., 331, 286 (Paper VIII)

Scardia, M., Prieur, J.-L., Pansecchi, L., Argyle, R.W., Sala, M., 2011, Astron. Nach., 332, 508 (Paper X)

Scardia, M., Prieur, J.-L., Pansecchi, L., Argyle, R.W., Spanó, P., Riva, M., Landoni, M., 2013, MNRAS, 434, 2803 (Paper XII)

Söderhjelm, S., 1999, A\&A 341, 121

Struve, F.G.W., 1827, "Catalogus Novus Stellarum Duplicium et Multiplicium", Typis J.C. Schuenmanni Typographi Academici, Dorpat

Struve,F.G.W.,1837, "Stellarum duplicium et multiplicium mensurae micrometricae..." ex Typographia Academica, Petropolis

Struve, O., 1843, "Catalogue de 514 étoiles doubles et multiples", Académie Impériale des Sciences, St. Petersbourg

van Leeuwen, F., 2007, "Hipparcos, the new reduction of the raw data", Springer Netherlands Ed.

Wilson, R.H., 1933, AJ 43, 41

Wilson, R.H., 1935, AJ 44, 71 